\title{
Halacaridae (Acari) von der Atlantikküste des borealen Nordamerikas
}

\author{
Ökologische und tiergeographische Faunenanalyse
}

\author{
I. Bartsch \\ Biologische Anstalt Helgoland (Zentrale); \\ Notkestraße 31, D-2000 Hamburg 52, Bundesrepublik Deutschland
}

\begin{abstract}
Halacaridae (Acari) from the Atlantic coast of boreal North America. Ecological and zoogeographical analysis. 38 halacarid species from the coasts of the boreal West Atlantic Ocean have been recorded. In a wide-spread net of stations, samples were taken from different substrata in marine and brackish waters in order to obtain information on the biology and ecology of halacarid species. Several habitats with their flora, fauna and halacarid species are described. In intertidal areas on the coasts of boreal North America fewer species were found than known from European coasts. Similar habitats on the west and east coasts of the North Atlantic Ocean are compared with respect to their halacarid population. In the rhombognathine and the genus Halacarellus, most species found in the West Atlantic Ocean are known in the East Atlantic too, living in similar habitats. The genera Anomalohalacarus and Copidognathus are common both in North American and European waters, but comparable biotopes are inhabited by different species, though often related or very similar in their appearance. $45 \%$ of the halacarid species found in the boreal West Atlantic Ocean are also known in the East Atlantic. Hypotheses as to the dispersal and geographical distribution of halacarid genera and species are discussed. It is supposed, that many of the amphiatlantic species invaded biotopes on the American and European coasts, shortly after these continental plates drifted apart.
\end{abstract}

\section{EINLEITUNG}

Die ersten umfassenden Kenntnisse der Halacariden-Fauna Nordamerikas brachte die Arbeit von Newell (1947). In dieser Arbeit werden für die Ostküste Nordamerikas 39 Arten genannt; davon treten 18 im borealen Gebiet zwischen Kap Hatteras und Neufundland auf. Erst drei Jahrzehnte später wurde erneut die Halacariden-Fauna an der Ostküste der USA untersucht (Bartsch, 1979b). Inzwischen sind aus dem Eulitoral und flachen Sublitoral der borealen Zone 38 Arten bekannt (Newell, 1947; Bartsch, 1976a, 1979b, 1980, 1981a) (vgl. Tab. 1).

Newell (1947) geht nur wenig auf Biologie und Ókologie der Halacariden-Arten ein. Bei eigenen Untersuchungen während eines sechswöchigen Aufenthalts in Rhode Island (vom 24. 4.-31. 5. 1978) wurde durch Probenentnahme aus verschiedenen Lebensräumen, Gezeitenbereichen, Salinitätszonen und Substrattypen versucht, einen Einblick in die ökologischen Ansprüche der einzelnen Arten zu erhalten. 


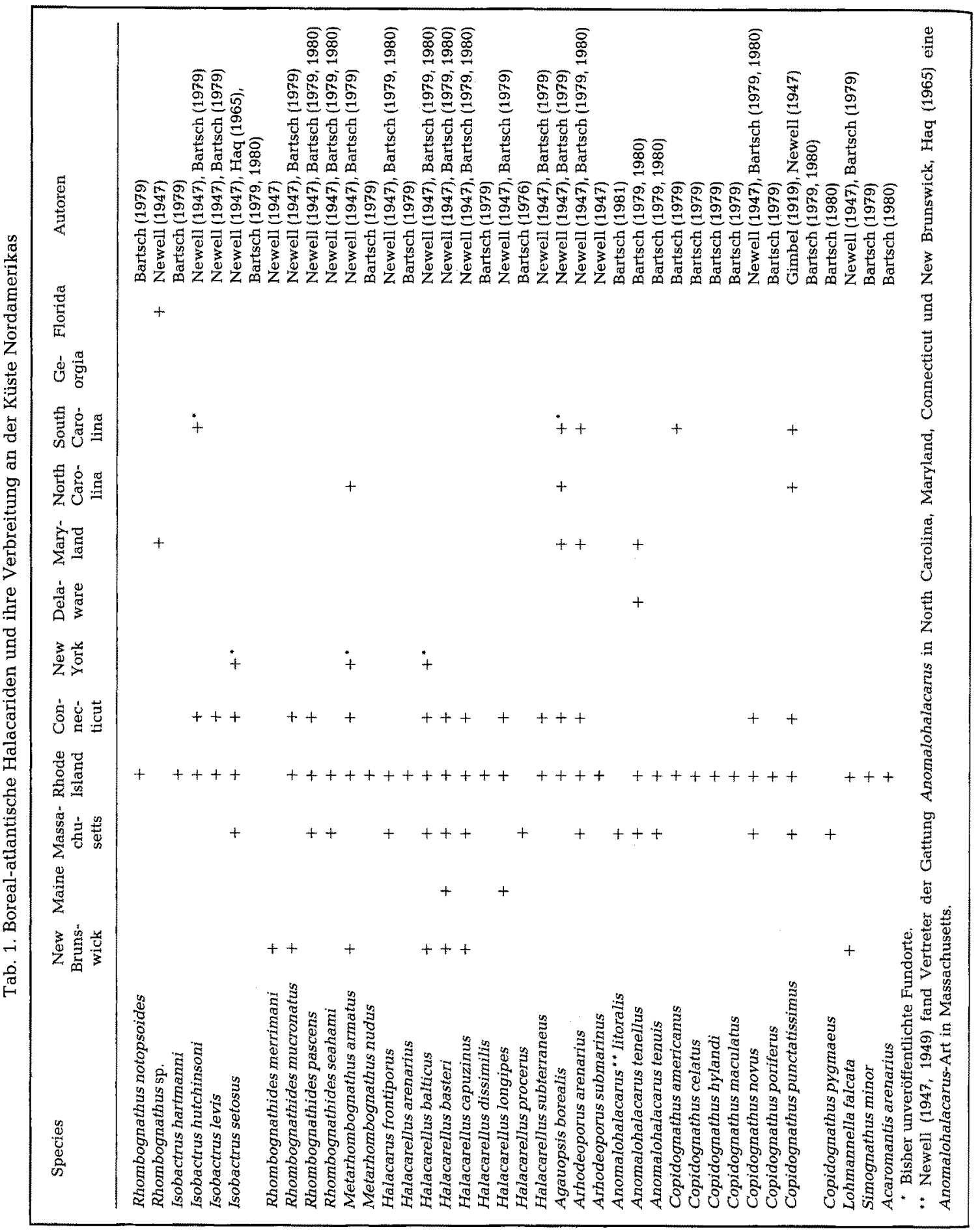




\section{PROBENENTNAHME UND -BEARBEITUNG}

Die Probenentnahme erfolgte stets zur Niedrigwasserzeit. Auf Hartsubstrat wurde der tierische und pflanzliche Bewuchs abgekratzt, bei Weichsubstrat wurden die oberen Schichten von 1 bis $5 \mathrm{~cm}$ abgetragen. Bei quantitativ auszuwertenden Proben wurde je nach Substrat eine abgesteckte Fläche oder ein bestimmtes Volumen eingesammelt. Parallel hierzu wurden Proben zur Bestimmung abiotischer Umweltfaktoren genommen.

Zur Anreicherung der Meiofauna wurden große Algenthalli unter kräftigem Wasserstrahl gespült und das Spülwasser durch ein Sieb (60 $\mu \mathrm{m}$ Maschenweite) gegossen. Sedimentproben wurden mehrmals - bis $20 \mathrm{mal}$ - mit reichlich Wasser aufgeschlemmt, anschließend dekantiert. Aufwuchsproben und Salzwiesenböden wurden in einen Siebsatz gebracht und mit kräftigem Wasserstrahl umspült. Das obere Sieb des Siebsatzes hatte eine Maschenweite von $1000 \mu \mathrm{m}$, das untere eine von $60 \mu \mathrm{m}$. Halacariden und andere Hartfauna-Gruppen überstehen den Spülvorgang ohne Schäden; bei der Weichtierfauna dagegen muß mit Verlusten gerechnet werden.

Ein Teil der Proben wurde sofort, unfixiert, durchgemustert, die Abundanz der einzelnen Tiergruppen und Halacariden-Arten geschätzt. Die Mehrzahl der Proben wurde in $70 \%$ Alkohol fixiert und je nach Fragestellung qualitativ oder quantitativ ausgewertet.

Der Inhalt im oberen Siebsatz wurde ebenfalls durchgemustert und Repräsentanten der Makrofauna zur Bestimmung dieser herausgesammelt. Da in der Fauna der Ostküste der USA viele Tiergruppen nur unzulänglich bearbeitet sind, wurde bei der Charakterisierung der Biotope oft auf die Art-Nennung verzichtet.

Der Salzgehalt wurde mittels Leitfähigkeitsmessung bestimmt, Temperatur mit einem Quecksilberthermometer $\left( \pm 0,5^{\circ} \mathrm{C}\right)$. Der Gehalt organischer Substanz in den Sedimentproben wurde durch Glühen, $5 \mathrm{~h}$ bei $560^{\circ} \mathrm{C}$, erhalten. Das Bestimmen der Korngrößenfraktionen erfolgte mit einem Siebsatz mit den Maschenweiten 0,63,90, $125,180,250,355,500,700,1000$ und $2000 \mu \mathrm{m}$.

\section{DAS UNTERSUCHUNGSGEBIET}

Das Untersuchungsgebiet liegt im Bereich von $41^{\circ} \mathrm{N}$ und $71^{\circ} \mathrm{W}$. Es erstreckt sich entlang der Küste Rhode Islands von Wickford am Narragansett Bay über Point Judith zu Stränden am Block Island Sound und schließt die dem Festland vorgelagerte Insel Block Island ein (Abb. 1). In diesem Gebiet finden sich exponierte Felsküsten, Strände bedeckt mit Kies, Geröll und Felsbrocken, Buchten mit sortierten, mittelfeinen Sanden, Brackwassertümpel mit nur schmalen Verbindungsgräben zum Meer, Aestuare und Salzwiesen. Der mittlere Gezeitenhub im Narragansett Bay beträgt je nach Lage 1,1 bis 1,4 m (Kremer \& Nixon, 1978). Die Wassertemperaturen im Narragansett Bay steigen im allgemeinen von $-0,5^{\circ} \mathrm{C}$ im Winter (Februar) bis $24^{\circ} \mathrm{C}$ in den Sommermonaten (Juli, August) (Kremer \& Nixon, 1978); in flachen Küstenabschnitten aber treten erheblich höhere Temperaturen auf. Der Salzgehalt am Eingang des Narragansett Bay schwankt zwischen 29 und $33 \%$ (Hicks, 1963); in Höhe der Station A 6 wurden 1957/58 Werte von 27,5 bis $32,4 \% \mathrm{~S}$ erhalten (Hicks, 1963). In Buchten und Aestuaren, im Mündungsbereich kleiner Bäche herrschten periodisch Süßwasserbedingungen. Einen Überblick über Geologie, Hydrographie, Meeresfauna und -flora der Küste des borealen Nordame- 


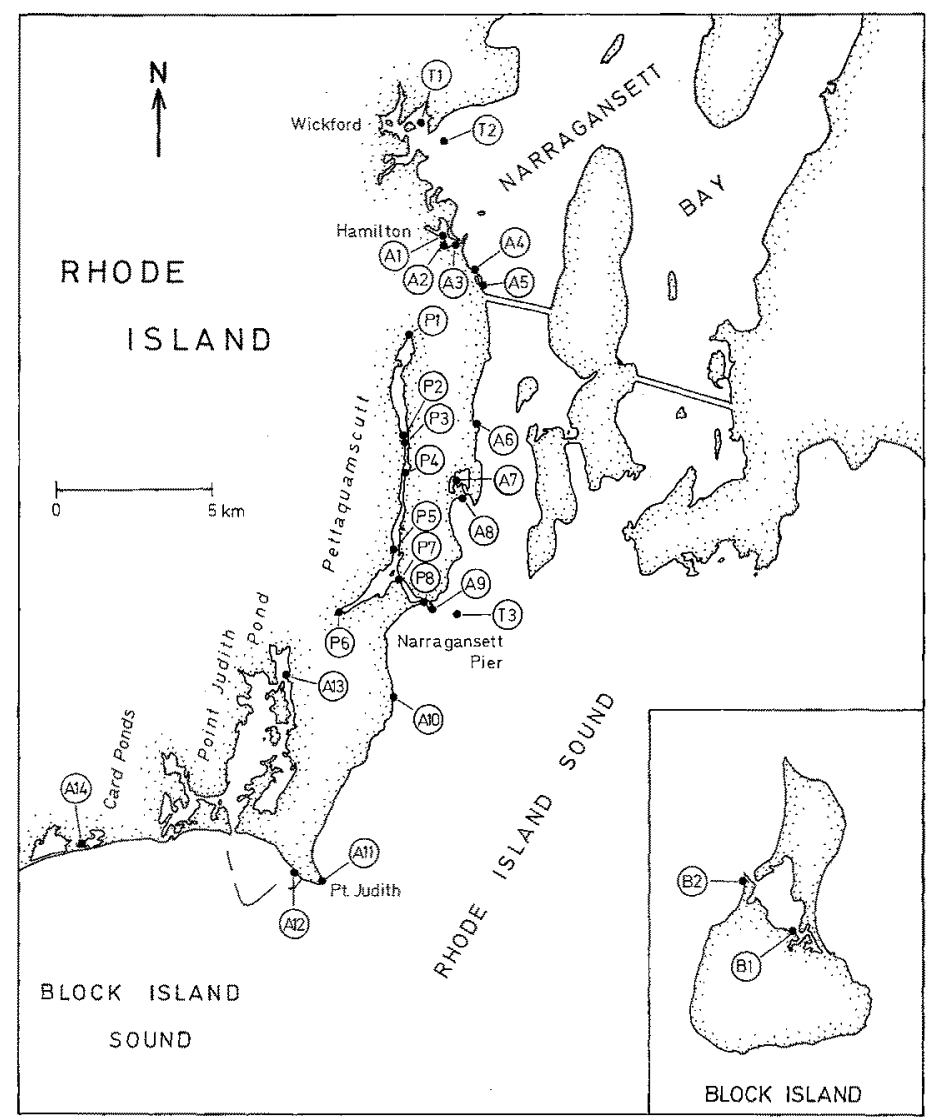

Abb. 1. Lage des Untersuchungsgebietes und der Stationen

rikas geben Milliman (1973), Pratt (1973) und Halvorson \& Dawson (1973). Über Strömungsverhältnisse, Bodenbeschaffenheit und Bodenfauna im Bereich des Narraganset Bay informieren Stickney \& Stringer (1957), Collins (1976) und McCaffrey et al. (1980) Einer der Zuflüsse des Narragansett Bay, nämlich der Pettaquamscutt, und seine Meio. fauna wurde von Kraus (1980) untersucht.

\section{Hydrographie und Substrate}

\section{Pettaquamscutt - Stationen $P 1$ bis $P 8$}

Der Pettaquamscutt ist ein fördeähnlicher Fluß mit nur schmaler Mündung in der Narragansett Bay. Der tidebeeinflußte Bereich reicht fast $8 \mathrm{~km}$ landeinwärts. Zahlreicht Bäche und Rinnsale ergießen sich auf dieser Strecke in den Fluß.

$\mathrm{Station} \mathrm{P1.} \mathrm{Der} \mathrm{schmale} \mathrm{Fluß} \mathrm{geht} \mathrm{über} \mathrm{in} \mathrm{die} \mathrm{tidebeeinflußte} \mathrm{Förde.} \mathrm{Zur} \mathrm{Zeit} \mathrm{de}$ Probenentnahmen wurden Salzgehaltswerte von maximal 0,6\% gemessen, doch deuteı 
Aufwuchs und Fauna darauf hin, daß auch höhere Werte auftreten. Das Sediment im Bereich der Wasserlinie war mit einer feinen Schicht flockigen Detritus bedeckt. Auf Steinen wuchsen in und unterhalb der Wasserlinie Balaniden (Balanus improvisus).

Station P 2. An der Mündung eines kleinen Rinnsales am Westufer des Pettaquamscutt. Das Ufer des Pettaquamscutt war sandig, im Hochwasserbereich von einzelnen Grasbüscheln durchwurzelt. Hier wurden Salzgehalte von 11,3-14,6\% gemessen. Das Sediment am Ufer des kleinen Nebenflusses war wesentlich detritusreicher $19,4 \%$ organische Substanz), im Bereich der Wasserlinie dicht mit Grassoden bewachsen. Der Salzgehalt in den stets wassergesättigten Grasbüscheln lag unter 0,5\%.

Station P 3. Ufer des Pettaquamscutt bei Bridgetown. Im Bereich und unterhalb der Niedrigwasserlinie siedelten auf Steinen und Felsbrocken der Uferbefestigung Balaniden (Balanus eburneus), Bryozoen (Membranipora) und Grünalgen (Enteromorpha Monostroma). Im mittleren und oberen Gezeitenbereich fehlte ein Aufwuchs fast völlig. In einer Bucht südlich der Brücke liegt ein Salzwiesengebiet. Das Sediment des Puccinellietums bestand bis zu $60 \%$ aus organischer Substanz. An den Salzwiesenkanten wuchs Enteromorpha. Im Bereich der Brücke betrug der Salzgehalt, je nach Wasserführung, 6,6-17,2\%. Am Ufer des Salzwiesenareals herrschten Salinitätsbedingungen von $11,1-16,3 \%$.

Station P 4. Kleiner Sandstrand an der Ostseite des Pettaquamscutt. Im mittleren und oberen Hang war der Strand mit grobem bis mittelfeinem, detritusarmen $(0,6 \%$ organische Substanz) Sand bedeckt; im unteren Hang hatte sich feines, detritusreiches Sediment abgelagert. Im mittleren Hang trat Grundwasser aus. Im Bereich der grundwasserführenden Schicht betrug der Salzgehalt 1,9\%; im Pettaquamscutt dagegen wurden 12,7-14,6\%o gemessen.

Station P 5. Pettaquamscutt bei Middle Bridge. An Steinen der Uferbefestigung und des Brückendammes wuchsen Balaniden, Enteromorpha, Monostroma und Fucus vesiculosus. In Stromnähe lag am Fuße des Brückendammes unsortiertes, recht grobes Sediment. Nördlich und südlich der Brücke bilden schmale Salzwiesenstreifen das Ufer des Pettaquamsacutt. Im Bereich der Brücke wurden Salzgehalte von $16,0-26,3 \%$ gemessen.

Station P 6. Salzwiesengebiet (Puccinellietum) durchflossen von einem kleinen Bach. Im Bach nimmt der Salzgehalt von der Mündung in den Pettaquamscutt zum Land hin rasch ab; im Laufe von wenigen Metern sank er von 22,6 auf $0,6 \%$ o. Auch innerhalb einer Tide sind die Salzgehaltsänderungen erheblich; an der Mündung herrschten je nach Tide Salzgehalte von 2,8-22,6\%.

Station P 7. Brücke über den Pettaquamscutt. An den Brückenwänden fand sich kurz unterhalb der Niedrigwasserlinie ein schmaler Aufwuchsstreifen, bestehend aus kleinen, büscheligen Algen, Hydrozoen-Stöcken, Polychaeten-(Fabricia-) und Amphipoden-(Corophium-)Röhren und kleinen Muscheln (Mytilus). Auf Steinen der Uferbefestigung wuchsen Grünalgen (Enteromorpha), Braunalgen (Fucus vesiculosus) und Balaniden (Semibalanus balanoides). Zwischen den Steinpackungen war das Ufer mit detritusreichen, groben, unsortierten Sedimenten bedeckt. In einer Bucht südlich der Brücke erstreckt sich ein Salzwiesengebiet (Puccinellietum), durchzogen von kleinen, vom Land her mit Süßwasser gespeisten Prielen. Im Brückenbereich wurden Salzgehaltswerte von 23,0-28,7\% gemessen. In den Prielen der Salzwiesen herrschten je nach Tide und Wasserführung Süß- oder Brackwasserbedingungen $(<0,5-18,7 \%$ \%). 


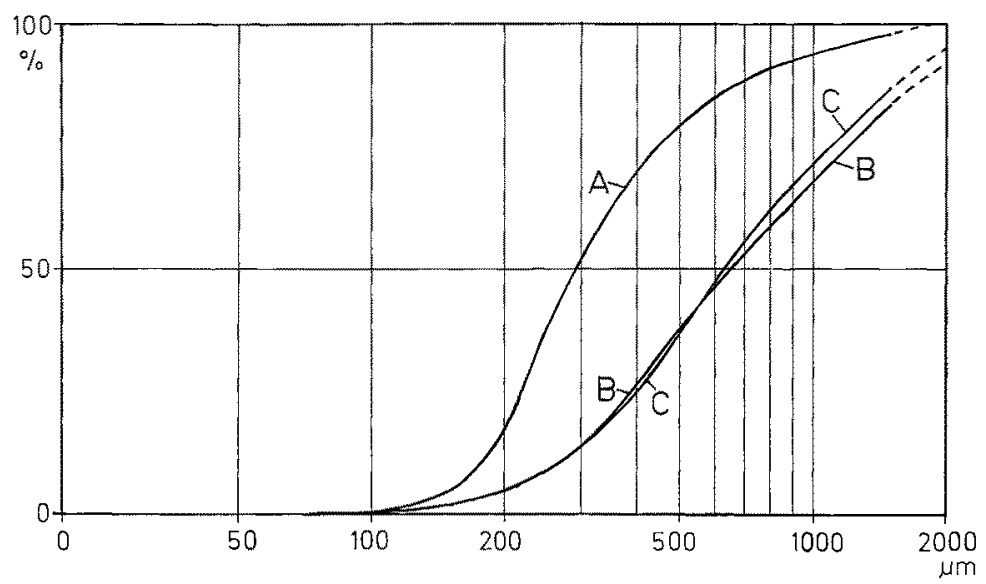

Abb. 2. Korngrößenverteilung, Station P 8. (A) Sediment unterhalb des Spülsaumes; (B) Sediment aus dem mittleren Hang; (C) Sediment aus dem unteren Hang

Station P 8. Sandstrand am nördlichen Ufer des Pettaquamscutt, daneben eine Steinpackung. Im Pettaquamscutt wurden Salzgehalte von $23,0-30,4 \%$ gemessen; im Sediment herrschten oft niedrigere Werte. Der Gehalt an organischer Substanz im Sediment war mit 0,57-0,66\% niedrig. Das Sediment am Hang war schwach sortiert (Abb. 2). Die Steinpackung war mit Balaniden überkrustet.

\section{Bucht bei Hamilton - Stationen A 1 bis A 3}

In der Bucht dehnt sich ein flacher Brackwassersee aus, mit nur schmaler Verbindung zum Narragansett Bay. In der Bucht herrscht, bedingt durch Süßwasserzuflüsse, ein wesentlich niedrigerer Salzgehalt als im Narragansett Bay.

Station A 1. Flacher Sandstrand und Salzwiesengebiet. Das Sediment am Strand enthielt in und unterhalb der Niedrigwasserlinie 1,2\% organische Substanz. Die oberste Sedimentschicht war oxidiert, erst in zwei bis drei Zentimeter Tiefe begann eine schwarze $\mathrm{H}_{2} \mathrm{~S}$-haltige Schicht. Im mittleren und oberen Strandbereich war das Sediment gröber, unsortiert (Abb. 3), es enthielt 0,9-1,1\% organische Substanz. Kiesel und Steine waren von Enteromorpha überwuchert. Im Salzwiesengebiet wuchs Spartina, dazwischen saßen Modiolus demissus, Enteromorpha- und Fucus vesiculosus-Büschel. Im oberen, sandigen Gezeitenbereich stand Queller (Salicornia) und Spergularia. An Station A 1 wurde zur Hochwasserzeit $18,9 \% \mathrm{~S}$ gemessen, zur Niedrigwasserzeit $5,5-14,0 \% \mathrm{~S}$.

Statio n A 2. In der Ecke der Bucht mündet ein zur Niedrigwasserzeit zwei bis drei Meter breiter, schnellfließender Bach in den Brackwassersee. Im Bachbett liegen Steine und Felsbrocken. Im Mündungsbereich des Baches waren Steine in und oberhalb der Niedrigwasserlinie mit Balaniden (Semibalanus balanoides, Balanus eburneus) und Grünalgen bewachsen. In einem Utbergangsbereich von nur wenigen Dezimetern verschwanden Brackwasseralgen und wurden durch eine Süßwasserflora ersetzt. Zur Hochwasserzeit wurden in Höhe der Brackwasserflora und -fauna Salzgehaltswerte von $15,5 \%$ gemessen; zur Niedrigwasserzeit waren Balaniden und Grünalgenpolster von Süßwasser $(<0,5 \%$ ) umspült; in den Algenpolstern aber herrschten noch oligohaline 


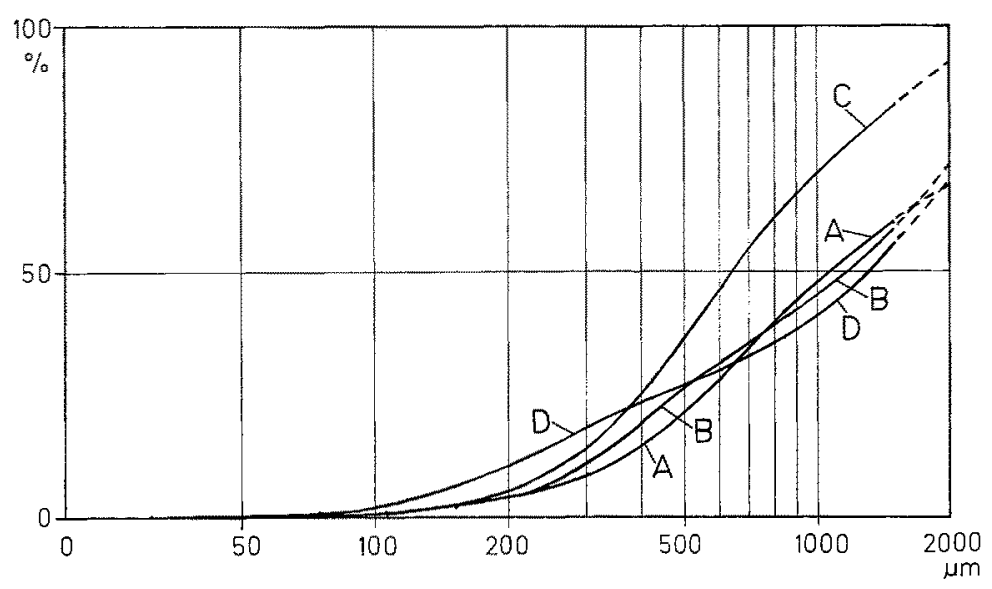

Abb. 3. Korngrößenverteilung, Stationen A 1 und A 2. (A) Station A 1, Sediment aus dem oberen Hang; (B) Station A 1, Sediment aus dem mittleren Hang; (C) Station A 1, Sediment aus dem Sandwatt; (D) Station A 2, Sediment aus dem oberen Hang

Bedingungen (0,5-0,6\% S). Am nördlichen Ufer grenzte ein geschlossenes Salzwiesengebiet (Puccinellietum) an, am Südufer ein mit einzelnen Spartina-Pflanzen bewachsener und mit grobem (Abb. $3 \mathrm{D}$ ), detritusreichem (2,1\% organische Bestandteile) Sediment bedeckter Sandhang. Zur Hochwasserzeit stand mesohalines Brackwasser (bis $22,8 \%$ S) über dem Hang, zur Niedrigwasserzeit sickerte oligohalines Brackwasser $(0,9 \% \mathrm{~S})$ aus dem mittleren Hang aus.

Station A 3. Langgestreckter, flacher Strand. Im unteren Tidenbereich war der Strand mit feinem, sehr detritusreichem Sediment bedeckt; im mittleren und oberen Hang wuchsen Spartina und Salicornia im detritusreichen, aber groben Sediment. Einzelne am Strand und im flachen Wasser liegende Felsbrocken waren mit Balaniden (Semibalanus balanoides) überzogen. Zur Niedrigwasserzeit herrschte ein Salzgehalt von $21,0 \%$. Im Sediment aber wurden $12,6 \%$ S gemessen.

\section{Ostküste Rhode Islands - Stationen A 4 bis A 11}

St a ti on A 4. Sandstrand nördlich der Brücke nach Jamestown. Den mittleren Hang bedeckt sortiertes, mittelfeines Sediment mit $0,7 \%$ organischen Bestandteilen. Im Bereich der Niedrigwasserlinie war das Sediment etwas feiner. Das Wasser im Narragansett Bay hatte zur Zeit der Probenentnahmen 25,6-27,9\% S.

Station A 5. Strand nördlich der Brücke nach Jamestown. Der Strand war mit unsortierten Sedimenten bedeckt. Im unteren und mittleren Gezeitenbereich hatte sich, in Flecken, Spartina ausgebreitet. Zwischen den Spartina-Wurzeln saßen Muscheln (Modiolus demissus); auf dem durchwurzelten Sediment wuchsen Balaniden, Grünalgen und Fucus vesiculosus. Auf größeren Felsbrocken bildeten Balaniden (Semibalanus balanoides) dichte Kolonien. Im Küstenwasser wurden Salzgehaltswerte von 25-28\% gemessen. Das Porenwasser im Sediment stand unter Süßwassereinfluß; hier herrschten Werte von $14-15 \%$.

Stati on A 6. Strand unterhalb des Marine Laboratory der Universität Rhode Islands. Zwischen Abschnitten mit Sandstrand bieten Steinpackungen Algen und Balaniden ein 
Substrat zum Siedeln. Ablaufendes Wasser hatte einen Salzgehalt von 25,6-26,3\%; auflaufendes einen von $30,9 \%$.

Station A 7. Wesquage Pond, ein Brackwassersee der nur über einen schmalen Graben mit dem Narragansett Bay in Verbindung steht. Zur Zeit der Probenentnahmen betrug der Salzgehalt 4-5\%.

Station A 8. Bucht mit Sandhang, mit mittleren, sortierten Sedimenten. Im Wasser der Bucht wurden 28,6\% $S$ gemessen.

Station A 9. Felsecke bei der Mündung des Pettaquamscutt. Zwischen Felsen liegen Strandabschnitte mit Felsbrocken, Geröll, Kies und Sand; daneben finden sich Fels- und Strandbecken, in denen während der Niedrigwasserphase Wasser stehen bleibt. Auf Felsen und in Felstümpeln standen Balaniden (Semibalanus balanoides), Grün-, Braun- und Rotalgen (Enteromorpha, Chaetomorpha, Fucus spiralis, F. vesiculosus, Ascophyllum nodosum, Chondrus crispus, Gigartina). Das Wasser im Narragansett Bay hatte $29,4 \%$ S. Im Sandhang war durch austretendes Brackwasser der Salzgehalt in den oberflächlichen Sedimentschichten etwas geringer; $24 \% \mathrm{~S}$ wurden gemessen.

Station A 10. Wellenexponierter Felsen südlich von Narragansett Pier. Die Felsen waren im unteren und mittleren Eulitoral mit Balaniden-Krusten (Semibalanus balanoides) bedeckt. Im oberen Gezeitenbereich wuchsen kleine, wenig-zellige Grünalgen; zwischen schützenden Felsen auch Enteromorpha. In tiefen Rockpools standen Cladophora, Codium, Chondrus, Corallina und in dichten Klumpen Mytilus. Im Narragansett Bay und in den regelmäßig überspülten Felsbecken wurden $30,1 \% \mathrm{~S}$ gemessen, in den höher gelegenen Felsbecken $25,5 \% \mathrm{~S}$.

Station A 11. Point Judith. Am Strand liegen Geröll und Felsbrocken mit einer reichen Aufwuchsflora und -fauna. Im oberen Litoral hatten sich kurze Grünalgen, darunter Fucus spiralis angesiedelt; im mittleren Gezeitenbereich wuchsen Fucus vesiculosus, Ascophyllum nodosum und Balaniden (Semibalanus balanoides), in der unteren Zone Fucus vesiculosus, Ascophyllum nodosum, Porphyra und Semibalanus balanoides; an der Niedrigwasserlinie standen Laminaria, Chondrus, Gigartina, Corallina, Monostroma und Domantia. Zwischen den Felsbrocken lag Kies und Sand. Es wurden Salzgehaltswerte von $29,4-30,9 \%$ erhalten.

\section{Südküste Rhode Islands - Stationen A 12 bis A 14}

Station A 12. Steiler Sandstrand mit detritus-armem Sediment, daneben eine Steinpackung mit einem dichten Enteromorpha-Streifen im oberen Gezeitenbereich. Der Salzgehalt im Sediment war mit $22,8 \%$ etwas geringer als im freien Wasser (30-31\%o).

Station A 13. Salzwiesenstück im Nordteil des Point Judith Pond. Zur Zeit der Probenentnahme herrschte ein Salzgehalt von 16,7\%o. Im mittleren Gezeitenbereich wuchs Spartina, dazwischen standen Modiolus demissus und Enteromorpha.

Station A 14. Ein durch einen breiten Strandwall vom Meer getrenntes Seensystem. Bei den an mehreren Stellen durchgeführten Salzgehaltsmessungen wurden Werte von $0,7-2,8 \%$ ermittelt.

\section{Block Island - Stationen B 1 und B 2}

Station B 1. Strand an der Westseite der Insel, bedeckt mit Sand, Kies und einzelnen Felsblöcken. Auf den Felsblöcken saßen Balaniden; an der Niedrigwasserlinie 
standen Algenpolster (Monostroma, Chondrus, Corallina) und Mytilus. Im flachen Wasser wurden $29,4 \% \mathrm{~S}$ gemessen.

$\mathrm{Stati}$ o B 2. Hafenanlage im inneren Teil des Great Salt Pond. Ein mit Kies und Geröll bedecktes Wattgebiet, dessen Steine mit Balaniden (Semibalanus balanoides), Mytilus, Fucus vesiculosus, $F$. distichus und Chondrus überwuchert waren. Daneben liegt ein kurzer Sandhang mit sortiertem Sediment mittlerer Korngröße; zwischen den Sandpartikeln fand sich ein hoher Anteil zerriebener Holzteile. Im Inneren der Bucht grenzte ein kleines Salzwiesengebiet an. In der Bucht wurden 29,4 \% S gemessen, im Salzwiesengebiet $19,8 \% \mathrm{~S}$; das Sediment am Sandhang stand unter Einfluß stark ausgesüßten Brackwassers.

\section{Sublitoral - Stationen $T 1$ bis $T 3$}

Station $T$ 1. Bucht bei Wickford. In einer flachen, nur $3 \mathrm{~m}$ tiefen Bucht, fand sich mittelfeines, detritusreiches Sediment. Die Oberflächenschicht war oxidiert.

$\mathrm{Station} \mathrm{T} \mathrm{2.} \mathrm{Narragansett} \mathrm{Bay} \mathrm{abseits} \mathrm{Wickford.} \mathrm{Im} \mathrm{feinen} \mathrm{Sediment} \mathrm{in} \mathrm{6-7} \mathrm{m}$ Tiefe war nur die Oberfläche oxidiert. Größere über das Sediment hinausragende Gegenstände waren bedeckt mit Hydrozoen-, Bryozoen- und Balaniden-Kolonien.

Station T 3. Narragansett Bay in Höhe der Mündung des Pettaquamscutt. Das Sediment in $20 \mathrm{~m}$ Tiefe war mittelfein, detritusreich, die Oberfläche war oxidiert. In Schleppnetzfängen trat unter anderem in großen Mengen der Dekapode Libinia emarginata auf.

\section{Faunenübersicht}

Zur Charakterisierung der untersuchten Lebensräume wird im folgenden die jeweils angetroffene Fauna kurz skizziert. Die erhaltenen prozentualen Anteile der einzelnen Tiergruppen an der Gesamtfauna spiegeln dabei die Frühjahrssituation wider.

\section{Salzwiesen}

Im unbewachsenen Sediment der Salzwiesenpriele (Stationen P 3, P 7) änderte sich - je nach Salzgehalt - die Faunenzusammensetzung innerhalb weniger Meter. In einer quantitativ ausgewerteten Probe von Station P 3 wurden 1340 Individuen $/ 100 \mathrm{~cm}^{3}$ gezählt. Die Nematoden stellten $83 \%$ der Meiofauna; daneben wurden Turbellarien, Oligochaeten (Énchytraeiden), Copepoden, Ostracoden (Cyprideis u. a. Cytheraceen, Cypridaceen) und Milben der Gruppen Oribatei (Ameronothrus), Acaroiden, Mesostigmata und Halacariden gesehen. An Station P 7 traten zudem große Mengen an Foraminiferen auf.

Im stets feuchten Puccinellietum (Stationen P 3, P 6, P 7, A 1) erschien eine der der Salzwiesenpriele ähnliche Fauna, jedoch mit anderen Abundanzen. In einer Probe von Station P 7 nahmen die Nematoden nur $44 \%$ der Fauna ein, die Foraminiferen $33 \%$ und die Halacariden $11 \%$.

Die von Süßwasser umspülten Grassoden am Nebenfluß der Station P 2 beherbergten Nematoden, Copepoden, Oribatei (Trimaloconothrus) und Halacariden.

Im groben Sediment der Spartina-Salzwiesen (Stationen A 2, A 5, A 13) dominierten oft die Copepoden. In einer an Station A 5 gesammelten quantitativ ausgewerteten 
Probe hatten sie einen Anteil von $49 \%$ an der Gesamtfauna; Halacariden stellten $24 \%$, Nematoden $18 \%$, Oligochaeten (Enchytraeiden, Tubificiden) $7 \%$. In unbedeutender Anzahl wurden Turbellarien, Tardigraden, Insekten-Larven, Foraminiferen, Collembolen und Trombidiiden registriert.

\section{Detritusreiche, wassergesättigte Sedimente}

In oligohalinen Brackwasserregionen (Stationen P 1, P 6, A 7, A 14) enthielt das flockige stets wasserbedeckte Sediment sowohl Süß- als auch Brackwasserelemente. Turbellarien, Nematoden, Kinorhynchen, Oligochaeten (Naididen - Nais, Amphichaeta -, Tubificiden, Tardigraden (Macrobiotiden), Copepoden, Ostracoden (Cytheraceen, Cypridaceen), Cumaceen (Almyracumi proximoculi), Oribatei (Trimaloconothrus), Hydrachnellen, Limnohalacariden und marine Halacariden traten auf.

An den nur bei extremem Niedrigwasser freiliegenden Wattgebieten der Stationen A 1 und A 3 herrschte eine hohe Siedlungsdichte. Eine ausgezählte Probe von der Station A 1 enthielt 44556 Tiere $/ 1000 \mathrm{~cm}^{3}$. Die zahlenmäßig wichtigste Gruppe, die Nematoden, nahmen $81 \%$ der Meiofauna ein; in weit geringerer Abundanz erschienen Copepoden, Oligochaeten, Halacariden, Turbellarien, Ostracoden, Polychaeten, Archianneliden und Foraminiferen.

Im Sublitoral (Stationen T 1, T 3) lebte in oberflächlichen Detritusschichten und in dem Sediment, das sich im Körperfilz des Dekapoden Libinia emarginata verfangen hatte, eine artenreiche Meiofauna. Foraminiferen, Nematoden, Kinorhynchen, Polychaeten, Copepoden, Amphipoden (Paraphoxus) und Halacariden wurden registriert.

\section{Sortierte Sedimente}

Detritusarme Gezeitenstrände wurden an den Stationen P 4, P 8, A 4, A 5, A 6, A 8 , A 12 und B 2 untersucht. An Station P 4 wurden Halacariden nur im mittleren Hang in der grundwasser-führenden Schicht angetroffen; sie waren begleitet von Nematoden und Copepoden.

In Proben aus dem oberen und mittleren Hang der Station P 8 wurden 3200-7200 Individuen $/ 1000 \mathrm{~cm}^{3}$ gezählt. Foraminiferen, Turbellarien, Nematoden, Polychaeten (Stygocapitella), Oligochaeten (Enchytraeiden, Tubificiden), Copepoden, Ostracoden, Collembolen, Mesostigmata und Halacariden waren vertreten (Abb. 4 A, 4 B). Im unteren Hang fanden sich außer den oben genannten Tiergruppen viel Tardigraden (Batillipes) (Abb. 4 C).

In den Sedimenten der weiten Strände entlang des Narragansett Bay (Stationen A 4, A 5, A 6, A 8) war die Fauna meist artenreicher. Eine quantitativ ausgewertete Probe aus dem unteren Hang der Station A 4 enthielt 14675 Individuen $1000 \mathrm{~cm}^{3}$. Die Nematoden stellten 58 \% der Fauna; Oligochaeten (Enchytraeiden) und Copepoden traten ebenfalls in hoher Abundanz auf (20 bzw. $18 \%$ ). Daneben beherbergte das Sediment Foraminiferen, Turbellarien, Archianneliden, Polychaeten (Scoloplos), Tardigraden, Collembolen. Im Sediment der Station A 8 fiel der Dekapode Emerita auf. Im Sand aus dem oberen Hang der Station A 4 wurden 10053 Tiere $/ 1000 \mathrm{~cm}^{3}$ gezählt. Die wichtigsten Gruppen waren die Nematoden (34\%), Enchytraeiden (29\%) und Copepoden (19\%). Sehr hoch war auch der Anteil der Turbellarien und Archianneliden an der Gesamtfauna; daneben fanden sich Polychaeten (Stygocapitella), Collembolen und die Milbengruppen Mesostigmata, Trombidiiden, Halacariden. Die Fauna im mittleren Hang der Station B 2 
unterschied sich von der eben genannten vor allem durch die in Abundanz auftretenden Mystacocariden (Derocheilocaris). Im unteren Hang fielen die Archianneliden (Protodriloides) besonders auf.

An dem wellenexponierten Strand der Station A 12 war die Mesopsammalfauna zahlenmäßig etwas ärmer. In einer dem oberen Hang entnommenen und quantitativ ausgewerteten Probe wurden 7990 Individuen $/ 1000 \mathrm{~cm}^{3}$ gezählt. Nematoden stellten $47 \%$ der Fauna. Auffällig war die große Menge an Oligochaeten (Enchytraeiden), die. $33 \%$ ausmachten. Copepoden hatten einen Anteil von $12 \%$, Turbellarien einen von

A

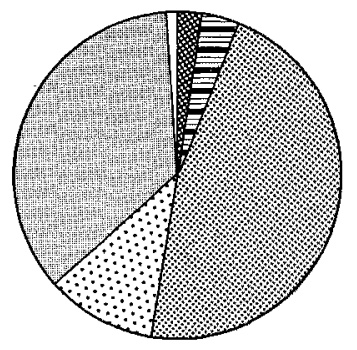

7196 Ind $/ 1000 \mathrm{~cm}^{3}$

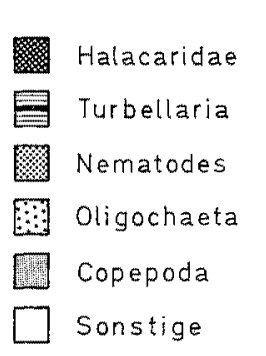

$\mathrm{B}$

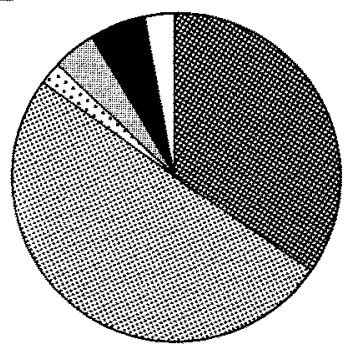

$3177 \mathrm{lnd} / 1000 \mathrm{~cm}^{3}$

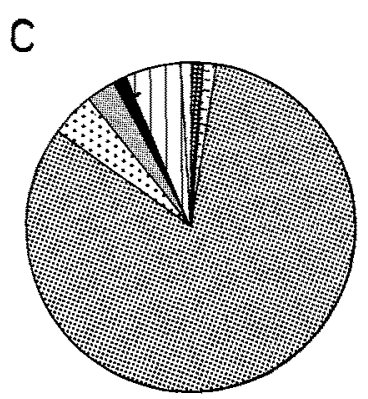

6680 ind. $/ 1000 \mathrm{~cm}^{3}$

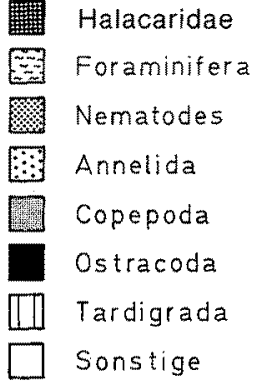

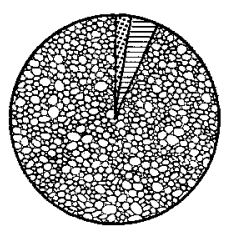

Metarhombognathus

Halacarellus

Anomalohalacarus



\section{Halacarellus}

Anomalohalacarus

Abb. 4. Prozentualer Anteil der Tiergruppen an der Gesamt-Meiofauna und prozentualer Anteil der Gattungen an der Halacariden-Fauna, Station P 8. (A) Sediment aus dem oberen Hang; (B) Sediment aus dem mittleren Hang; (C) Sediment aus dem unteren Hang 
$6 \%$; daneben wurden Collembolen und Halacariden angetroffen. Eine quantitativ ausgewertete Probe aus dem mittleren Hang enthielt 9988 Tiere $/ 1000 \mathrm{~cm}^{3}$. Wiederum dominierten die Nematoden $(40 \%)$, gefolgt von Copepoden $(33 \%)$, Oligochaeten (Enchytraeiden) ( $22 \%$ ), einigen Turbellarien, Gastropoden, Ostracoden, Amphipoden (Talorchestia), Collembolen und Halacariden. Im Niedrigwasserbereich waren die zahlenmäßig wichtigsten Gruppen die Archianneliden und Turbellarien; daneben wurden Nematoden, Oligochaeten, Ostracoden, Amphipoden, Tardigraden, Collembolen und Halacariden erkannt.

A

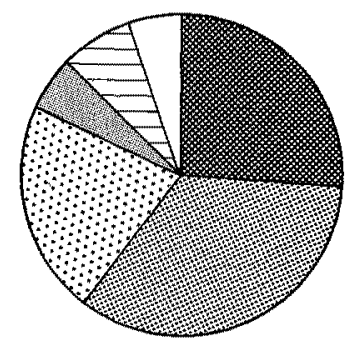

$3324 \mathrm{lnd} / 1000 \mathrm{~cm}^{3}$

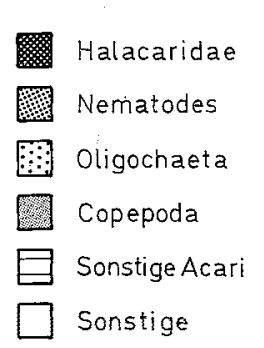

Sonstige

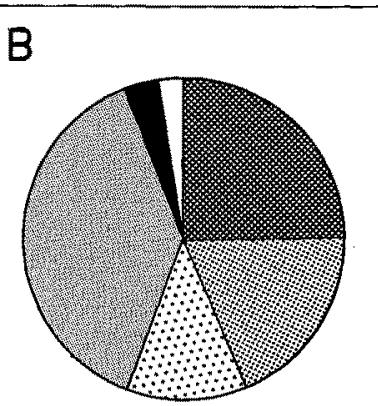

20574 ind. $/ 1000 \mathrm{~cm}^{3}$

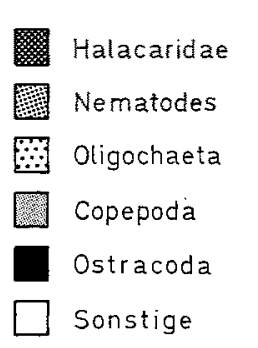

C



50690 ind $/ 1000 \mathrm{~cm}^{3}$

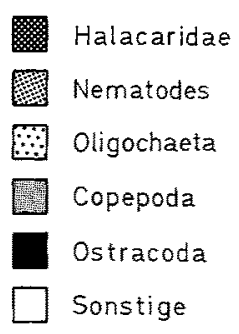

Sonstige

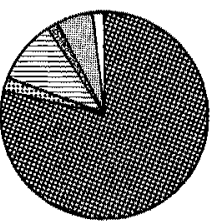

Rhombognathides

Metarhombognathus

Halacarellus

Agauopsis

[7. Arhodeoporus Sonstige

(1)

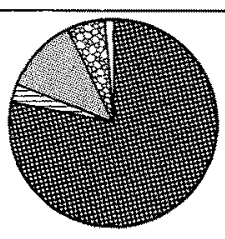

Rhombognathides

Halacarellus

Arhodeoporus

Anomalohal acarus

Sonstige

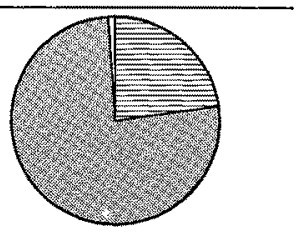

Halacarellus

Arhodeoporus

$\square$ Sonstige

Abb. 5. Prozentualer Anteil der Tiergruppen an der Gesamt-Meiofauna und prozentualer Anteil der Gattungen an der Halacariden-Fauna, Station A 1. (A) Sediment aus dem oberen Hang; (B) Sediment aus dem mittleren Hang; (C) Sediment aus dem Sandwatt 


\section{Unsortierte Sedimente}

An Station P 2 war die Fauna im durchwurzelten Sediment, gesammelt im Bereich der Hochwasserlinie, äußerst arm; einzelne Nematoden, Copepoden, Ostracoden und Insekten-Larven traten auf, dazwischen lebten terrestrische Milben (Mesostigmata, Rhodacariiden, Trombidiiden) und Halacariden.

Einige der an den Sandstränden der Bucht bei Hamilton entnommenen Proben wurden quantitativ ausgewertet. Im Sandwatt der Station A 1 wurden 50690 Individuen $/ 1000 \mathrm{~cm}^{3}$ gezählt. Die individuenreichste Gruppe waren die Nematoden, die $36 \%$ der Meiofauna stellten (Abb. 5 C), gefolgt von Halacariden (31\%) und Oligochaeten (Tubificiden) $(20 \%)$; in geringer Abundanz traten Foraminiferen, Turbellarien, Polychaeten, Copepoden, Ostracoden (Cytheraceen) und Cladoceren auf. Im mittleren Hang, bei einer Gesamtbesiedlung von 20574 Tieren $/ 1000 \mathrm{~cm}^{3}$, dominierten die Copepoden (39\%) (Abb. 5 B); es folgten mit $25 \%$ die Halacariden, mit $19 \%$ die Nematoden und mit $11 \%$ die Oligochaeten (in erster Linie Enchytraeiden), zudem Ostracoden, Polychaeten (Stygocapitella), Insekten-Larven, Collembolen, Foraminiferen, Turbellarien und terrestrische Milben. In einer Probe aus dem oberen Hang wurden 3324 Individuen/1000 $\mathrm{cm}^{3}$ gezählt. Die drei wichtigsten Tiergruppen waren Nematoden (34\%), Halacariden $(26 \%$ ) und Enchytraeiden (20\%) (Abb. 5 A). In geringerer Abundanz erschienen Copepoden, terrestrische Milben, Polychaeten (Stygocapitella), Collembolen, Pseudoscorpionen und Insekten-Larven. Auch kiesige, mit Queller bewachsene Strandabschnitte (Stationen A 1, A 3) beherbergten eine Fauna, wie in Abb. 5 A dargestellt.

Am Rhode Island und Block Island Sound (Stationen A 9, A 11, B 1) lebten in den groben Sedimenten im mittleren und unteren Gezeitenbereich Foraminiferen, Nematoden, Kinorhynchen, Nemertinen, kleine Mytilus, Polychaeten (Nereiden, Capitelliden), Oligochaeten (Tubificiden), Copepoden, Ostracoden, Isopoden (Jaera), Amphipoden (Elasmopus, Corophium, Unciola, Jassa), Ophiuroiden (Amphipholis) und Halacariden.

\section{Grünalgen-Thalli}

In den bei Ebbe von Süßwasser, bei Flut von Brackwasser umströmten Enteromorpha- und Monostroma-Polster an Station A 2 dominierten die Copepoden; sie hatten einen Anteil von 78-92\% an der Meiofauna. In den stark von Süßwasser beeinflußten

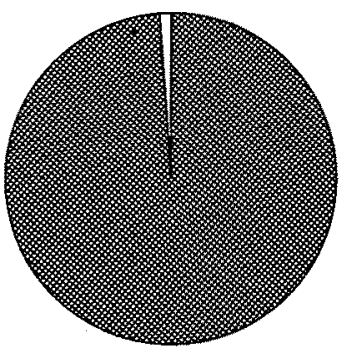

$374 \mathrm{ind} .10 \mathrm{~cm}^{2}$



Metarhombognathus

Sonstige

Abb. 6. Prozentualer Anteil der Halacariden an der Gesamtfauna und prozentualer Anteil der Gattung Metarhombognathus an der Halacariden-Fauna, Station A 12. Enteromorpha aus dem oberen Gezeitenbereich 
Algenpolstern $(<0,5 \% \mathrm{~S}$ ) stellten die Halacariden nur 0,4\% der Fauna, in Proben mit höherer Salinität $(0,5 \% \mathrm{~S})$ dagegen $18 \%$. Neben Copepoden und Halacariden bewohnten Oligochaeten (Nais), Ostracoden (Cytheraceen, Cypridaceen), Amphipoden (Gammarus), Chironomiden-Larven, Hyadesiiden (Hyadesia) und Oribatei die Algenpolster. Zwischen den Monostroma-Thalli der Stationen P 1 und P 3 hatten die Copepoden keine solch große Bedeutung wie an Station A 2.

Im mesomixo-, polymixo- und euhalinen Bereich fand sich in der oberen und mittleren Gezeitenzone oft ein breites Enteromorpha-Band (Stationen P 5, P 7, A 6, A 9, A 10, A 12). In den Proben dominierten stets die Halacariden. In quantitativ ausgewerte-



Abb. 7. Prozentualer Anteil der Tiergruppen an der Gesamt-Meiofauna und prozentualer Anteil der Gattungen an der Halacariden-Fauna, Station A 1. (A) Enteromorpha auf Sediment vom oberen Hang; (B) Enteromorpha auf Sediment vom unteren Hang

ten Proben von den Stationen A 6 und A 12 hatten sie einen Anteil von 73-99 \% der Gesamtmeiofauna (vgl. Abb. 6). Die Begleitfauna setzte sich zusammen aus Foraminiferen, Nematoden, kleinen Muscheln (Mytilus), Gastropoden, juvenilen Balaniden, Copepoden, Ostracoden, Tardigraden (Echiniscoides), Insekten-Larven und Hyadesiiden (Hyadesia). Zwischen sehr groben Enteromorpha-Strängen, gesammelt im unteren Eulitoral, erschienen außer den bereits erwähnten Meiofaunagruppen auch Isopoden (Jaera) und Amphipoden (Gammarus). Enteromorpha-Proben aus flachen Felstümpeln enthielten in hoher Abundanz Hyadesiiden (Hyadesia) und in geringen Mengen Oribatei (Hermannia, Ameronothrus) und Halacariden. 
Eine andere Fauna wurde zwischen Enteromorpha-Polster auf Salzwiesenböden angetroffen. An den Salzwiesenkanten der Stationen P 3 und P 6 lebten in dem Substrat aus Enteromorpha und Sediment Nematoden, Enchytraeiden, Copepoden, Ostracoden (Cytheraceen), Dipteren-Larven, Oribatei (Ameronothrus, Trimalonothrus) und Halacariden. In meso- und polyhalinen Gewässerabschnitten (Stationen P 5, P 7, A 1) standen an den Salzwiesenkanten außer Enteromorpha auch Fucus vesiculosus, Muscheln (Modiolus demissus) und Polychaeten (Fabricia sabella). In den oberflächlichen Salzwiesenschichten wurden außer den bereits genannten Gruppen auch Foraminiferen, Gastropoden, Tubificiden, Tanaidaceen (Leptochelia), Isopoden (Jaera), Amphipoden (Gammarus), Trombidiiden und Mesostigmata angetroffen.

Der Enteromorpha-Bewuchs der Steine und Kiesel, gesammelt am Gezeitenstrand der Station A 1, enthielt viele Sedimentbewohner. In einer Probe aus dem oberen Hang wurden 8800 Tiere $/ 1000 \mathrm{~cm}^{3}$ gezählt. Die Halacariden stellten $66 \%$ der Gesamtfauna (Abb. 7 A). Semiterrestrisch lebende Milben der Gruppen Trombidiiden, Hyadesiiden (Hyadesia) und Oribatei (Ameronothrus) nahmen $25 \%$ der Fauna ein. Daneben wurden einige Copepoden und Oligochaeten (Enchytraeiden) gefunden. Im unteren Hang war die Siedlungsdichte im Enteromorpha-Sediment wesentlich höher; 91320 Individuen/ $1000 \mathrm{~cm}^{3}$ wurden festgestellt. Die Halacariden, mit 17180 Tieren/1000 cm ${ }^{3}$, entsprechend $19 \%$ der Meiofauna, standen an vierter Stelle nach Copepoden, Ostracoden und Nematoden (Abb. 7 B).

Auf treibenden Ulva-Thalli (Station P 5) wurden Copepoden, Ostracoden (Cytheraceen), Isopoden (Idotea baltica), große Mengen von Gastropoden (Hydrobia) und Halacariden angetroffen.

\section{Braunalgentange}

Die Besiedlung der Fucus vesiculosus-Thalli in Salzwiesengebieten (Stationen P 5, A 1) war spärlich. In den Proben dominierten die Halacariden (Abb. 8), gefolgt von Gastropoden, Isopoden (Jaera), Oribatei (Ameronothrus), Oligochaeten, Dipteren-Larven und Copepoden.

In lenitischen Brackwasserregionen (Stationen P 5, P 7) wurde auf den im mittleren und unteren Eulitoral gesammelten Fucus vesiculosus-Büschel vor allem kleine Nematoden gefunden. In einer quantitativ ausgewerteten Probe (Station P 5) stellten sie mit
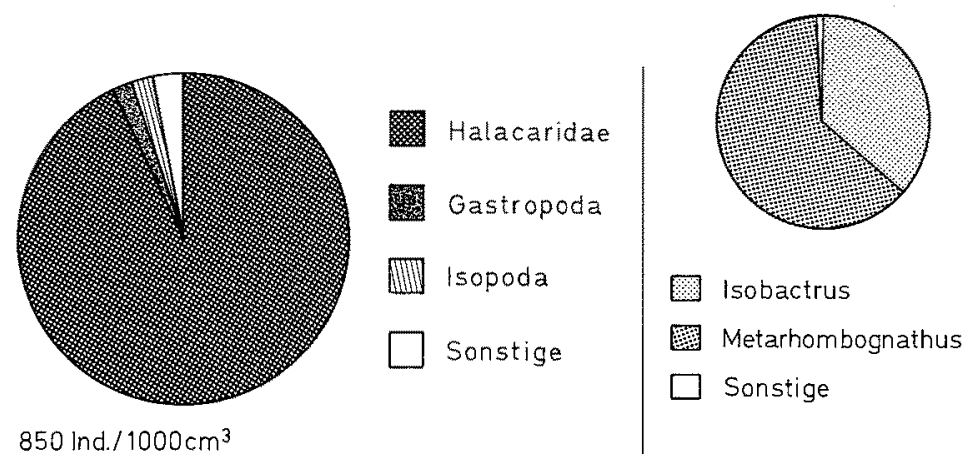

Abb. 8. Prozentualer Anteil der Tiergruppen an der Gesamt-Meiofauna und prozentualer Anteil der Gattungen an der Halacariden-Fauna, Station A 1. Fucus vesiculosus aus dem Salzwiesengebiet 
3162 Individuen $/ 1000 \mathrm{~cm}^{3} 50 \%$ der Fauna; die Copepoden machten $34 \%$ aus; daneben erschienen Foraminiferen, Ostracoden (Cytheraceen), Isopoden (Jaera), Amphipoden (Gammarus), Insekten-Larven und die Milben Mesostigmata, Hyadesiiden, Halacariden. An Station P 7 waren zudem die Foraminiferen in hoher Abundanz vertreten.

Auf den im oberen Litoral (Station A 11) gesammelten Fucus spiralis dominierten die Halacariden. In einer Probe wurden 1413 Halacariden $/ 1000 \mathrm{~cm}^{3}$ gezählt (Abb. 9 A). Unter den Büscheln hielten sich große Mengen. Amphipoden (Hyale) auf. Außerdem

A
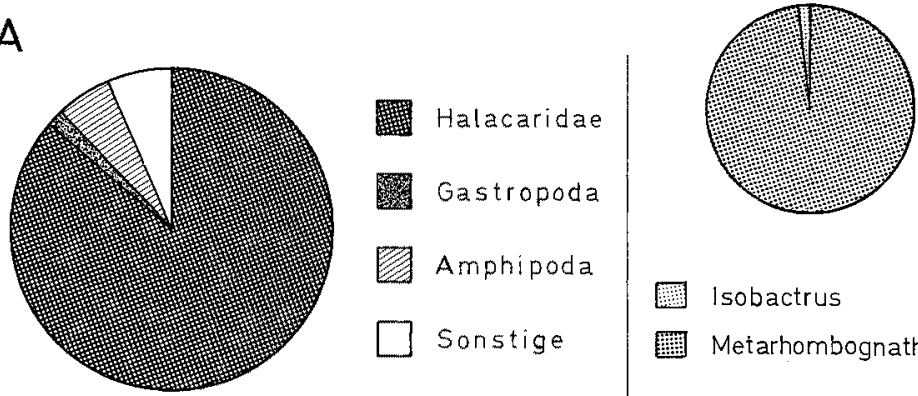

Isobactrus

Metarhombognathus

$1633 \mathrm{lnd} . / 1000 \mathrm{~cm}^{3}$

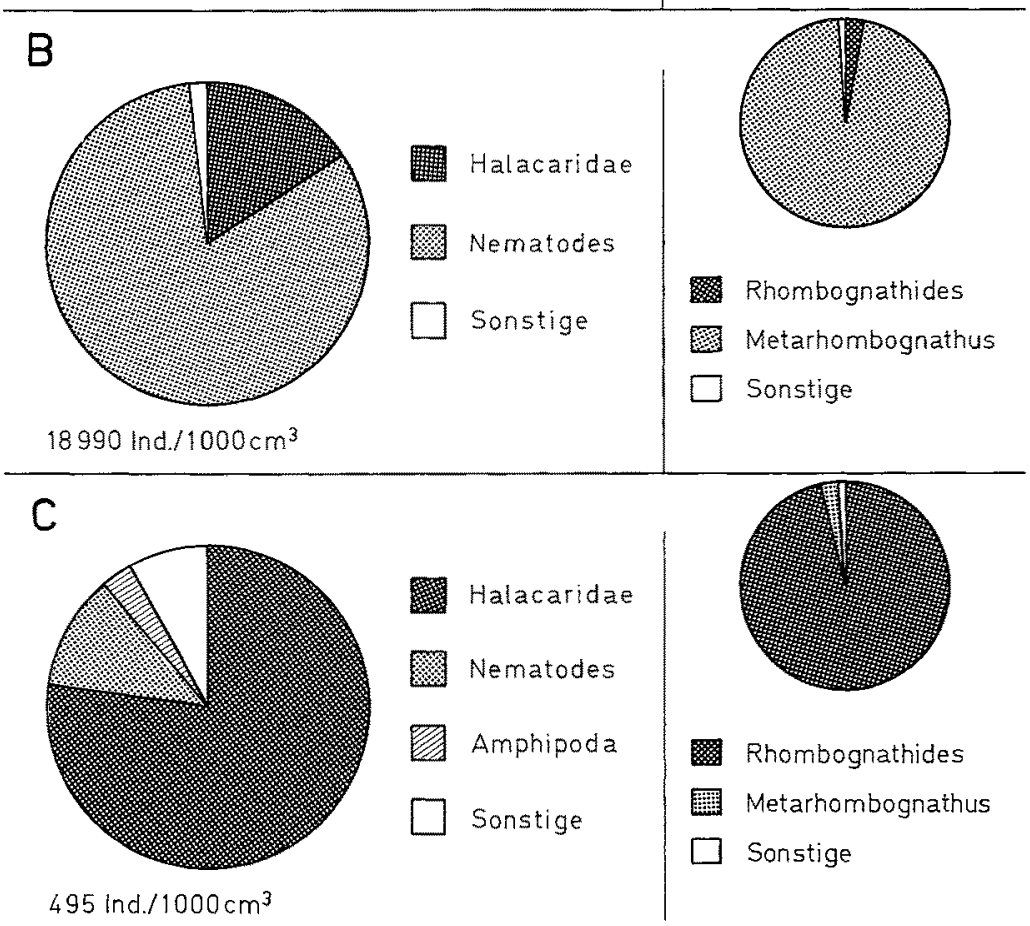

Abb. 9. Prozentualer Anteil der Tiergruppen an der Gesamtfauna und prozentualer Anteil der Gattungen an der Halacariden-Fauna, Station A 11. (A) Fucus serratus aus dem Hochwasserbereich; (B) Fucus vesiculosus aus dem mittleren Gezeitenbereich; (C) Ascophyllum nodosum aus dem unteren Gezeitenbereich 
wurden Foraminiferen, Nematoden, Polychaeten, Oligochaeten (Enchytraeiden), Copepoden, Tardigraden und Hyadesiiden in den Proben gefunden.

Die im mittleren und unteren Eulitoral (Stationen A 6, A 9, A 11, B 2) gesammelten, mit Epiphyten bewachsenen Fucus vesiculosus-Thalli enthielten eine wesentlich reichere Fauna. Die dominierende Tiergruppe waren stets die Nematoden. In den Proben von Station A 6 nahmen sie $81 \%$, von Station A $1183 \%$ der Fauna ein (Abb. 9 B). Die Halacariden stellten nur $16 \%$ der Fauna. Auf Fucus vesiculosus-Büschel mit geringem Epiphyten-Besatz wurden nur wenige Tiere gesehen; der Anteil der Nematoden war auf $50 \%$ und weniger gesunken.

Auf Ascophyllum-Thalli (Stationen A 9, A 11) war die Fauna spärlich. An Station A 11 dominierten die Halacariden; auf Thalli mit geringem Bewuchs stellten sie $77 \%$ der Fauna (Abb. 9 C), auf Thalli mit dichtem Bewuchs $70 \%$. Es folgten Nematoden, Amphipoden (Hyale, Gammarellus, Luconacia, Jassa), Bivalvier (Mytilus), Copepoden, Oligochaeten, Ostracoden, Isopoden (Idotea baltica), Foraminiferen, ChironomidenLarven, Hyadesiiden und Oribatei.

Laminaria-Thalli waren kaum besiedelt; nur einzelne Copepoden, Gastropoden, Amhipoden und Halacariden wurden gesehen.

\section{Kleine, verzweigte Algen und Tierkolonien}

An den Stationen A 6, A 9, A 11, B 1, B 2 fand sich in und unterhalb der Niedrigwasserzone, in Felstümpeln im Bereich der Wasserlinie, zwischen dichten Algenpolster (Monostroma, Enteromorpha, Chaetomorpha, Ulva, Chondrus, Gigartina, Ceramium, Corallina) und Laminaria-Rhizoiden stets ein dichter Bewuchs von Polychaeten- (Fabricia-) und Amphipoden (Corophium-JWohnröhren, Hydrozoen- und Bryozoen-Kolonien und Mytilus. Die Fauna in diesem Spaltensystem war stets arten- und individuenreich, so wurden an Station A 11 bis zu 114080 Tiere/1000 $\mathrm{cm}^{3}$ gezählt. In den im Niedrigwasserbereich gesammelten Proben stellten Halacariden 16-32 \% der Fauna, im Sublitoral dagegen war ihr Anteil unbedeutend. Neben Halacariden fanden sich Foraminiferen, Turbellarien, Nemertinen (Lineus), Nematoden, Polychaeten (Phyllodociden, Sylliden, Nereiden, Fabriciinen), Oligochaeten (Tubificiden), Gastropoden (Littorina), Bivalvier, Copepoden, Ostracoden, Tanaidaceen (Tanais), Isopoden (Idotea, Erichsonella, Jaera), Amphipoden (Gammarus, Gammarellus, Calliopius, Amphithoe, Corophium, Jassa, Luconacia), Insekten-Larven (Chironomiden, Clunioniden), Trombidiiden und Mesostigmata. In Brackwasser-beeinflußten Regionen (Station P 7) waren Flora und Fauna bedeutend artenärmer.

\section{Balaniden- und Muschel-Kolonien}

Die Fauna zwischen Balanus improvisus (Station P 1) war stark geprägt durch Süßwasserformen. Es traten die Tiergruppen Turbellarien, Nematoden, Copepoden, Ostracoden (Cypridaceen), Amphipoden (Gammarus), Chironomiden und Halacariden auf. An den erheblichen Salinitätsschwankungen ausgesetzten Krusten an Station A 2 lebten vor allem Halacariden, Hyadesiiden (Hyadesia), Oribatei (Ameronothrus), Chironomiden-Larven und Amphipoden (Gammarus).

Zwischen den Kolonien von Balanus eburneus und Bryozoen an Station P 3 war die Fauna artenreicher; angetroffen wurden viele Nematoden, einzelne Polychaeten (Nereis succinea), Oligochaeten (Naididen, Enchytraeiden, Tubificiden), Copepoden, wenig Ostracoden (Cytheraceen), Tanaidaceen (Leptochelia) und Halacariden. 
Semibalanus balanoides-Krusten (Stationen P 7, P 8, A 5) waren in erster Linie von Hyadesiiden (Hyadesia) bewohnt; daneben wurden einige Tardigraden (Echiniscoides) und Halacariden angetroffen. An den Stationen A 6, A 9, A 10, A 11 und B 2 fielen in den Balaniden-Proben (Semibalanus balanoides, Chthamalus fragilis), gesammelt im oberen und mittleren Gezeitenbereich, die großen Mengen an Turbellarien auf. In quantitativ ausgewerteten Proben stellten sie bis zu $44 \%$ der Fauna; die Halacariden dagegen nahmen nur $19 \%$ und die Nematoden $15 \%$ ein. Außerdem erschienen Gastropoden (Littorina), Oligochaeten (Enchytraeiden), Tardigraden und Hyadesiiden. Im unteren Eulitoral traten zudem Polychaeten (Fabricia), kleine Mytilus, Copepoden und Amphipoden (Corophium, Jassa) auf.

Zwischen Modiolus-Klumpen in Salzwiesen (Stationen A 1, A 5, A 13) siedelten Foraminiferen, Nematoden, Polychaeten (Fabricia sabella), Copepoden, Isopoden (Jaera), Amphipoden (Allorchestes) und Halacariden.

Dichte Mytilus-Bestände fanden sich in Felstümpeln der Station A 10. In diesem Habitat lebten außer den oben genannten Gruppen auch Nemertinen (Lineus), Ostracoden, Amphipoden (Gammarus, Hyale, Corophium), Ophiuroiden und ebenfalls Halacariden.

\section{DIE HALACARIDEN DER UNTERSUCHTEN LEBENSRÄUME}

Zur Beschreibung der Halacariden-Fauna wird die in Bartsch (1978) verwendete Unterteilung der Biotope übernommen. Untersuchungen an den Küsten Europas (Bartsch, 1972, 1974a) haben gezeigt, daß juvenile und adulte Halacariden denselben Lebensraum besiedeln; ein auf 6 Wochen begrenzter Untersuchungs-Zeitraum ist somit ausreichend, um einen repräsentativen Uberblick über die Halacariden-Fauna zu erhalten.

\section{Weichböden}

\section{Salzwiesen}

In Grassoden, umspült von oligohalinem Brackwasser und Süßwasser war Copidognathus poriferus zu finden.

Im höhergelegenen, lange Zeiten trockenliegenden Puccinellietum wurden nur selten Halacariden angetroffen; eine an diesen Lebensraum angepaßte Fauna fehlte.

Tab. 2. Halacariden-Arten im Sediment von Puccinellium- bzw. Spartina- und Salicornia-Salzwiesen

\begin{tabular}{|ll|}
\hline Puccinellium-Salzwiesen & Spartina- und Salicornia-Salzwiesen \\
\hline Halacarellus balticus & Isobactrus setosus \\
Copidognathus hylandi & Rhombognathides mucronatus \\
& Metarhombognathus armatus \\
& Halacarellus balticus \\
Halacarellus basteri \\
Halacarellus subterraneus \\
Agauopsis borealis \\
Copidognathus americanus \\
Copidognathus hylandi \\
\hline
\end{tabular}


Im unteren, stets feuchten Puccinellietum und in detritusreichen Sedimenten kleiner Salzwiesenpriele trat in großen Mengen Copidognathus hylandi auf, daneben erschienen einige Halacarellus balticus. Copidognathus hylandi stellte $97 \%$ und mehr der Halacariden-Fauna.

Spartina-Salzwiesen, mit teils groben, kiesigen, teils feinen Sedimenten beherbergten in den obersten Sedimentschichten (0-2 mm Tiefe) eine artenreiche Fauna (Tab. 2). In der Mehrzahl der untersuchten Biotope war die Sedimentoberfläche von feinen Algen überwuchert; entsprechend dominierten dann die Rhombognathinen. Die wichtigste Art in diesen Sedimenten war meist Rhombognathides mucronatus, der einen Anteil von :8-64\% der Hálacariden-Fauna einnahm; Metarhombognathus armatus und Isobactrus setosus, ebenfalls häufig angetroffen, stellten $8-45$ bzw. 0,2-62\% der Fauna.

Wassergesättigte, detritusreiche Sedimente

In Zonen mit oligomixohalinen Bedingungen lebten Copidognathus hylandi und C. poriferus. C. poriferus war selbst in schwach salzigem Süßwasser noch anzutreffen.

Im mesomixohalinen Bereich war die Halacariden-Fauna meist artenreicher. In hohen Abundanzen erschienen Arhodeoporus arenarius und Halacarellus capuzinus; zusammen stellten sie 83-100\% der Halacariden-Fauna, dabei entfielen auf $A$. arenarius $58-79 \%$ und auf $H$. capuzinus $21-27 \%$. In einer quantitativ ausgewerteten Probe wurde von diesen zwei Arten 1581 Individuen $/ 100 \mathrm{~cm}^{3}$ gezählt. In geringen Zahlen erschienen zudem Rhombognathus notopsoides, Isobactrus setosus, Rhombognathides mucronatus, Metarhombognathus armatus, Halacarellus subterraneus, Anomalohalacarus tenellus und Acaromantis arenarius.

In den sublitoralen Sedimenten dominierte Arhodeoporus submarinus. Daneben traten Copidognathus punctatissimus, C. novus, C. maculatus, C. celatus, Halacarellus dissimilis, $H$. basteri, $H$. longipes und Lohmannella falcata auf.

\section{Sandlückensystem}

In mittelfeinen, detritusarmen Sedimenten trat im oberen und mittleren Hang vor allem Anomalohalacarus tenellus auf, in geringen Mengen auch A. tenuis. Die Anomalohalacarus-Arten stellten oft 77-96\% der Halacariden-Fauna (vgl. Abb, 4); bis zu 106

Tab. 3. Halacariden-Arten des Sandlückensystems im Gezeitenstrand

\begin{tabular}{|ll|}
\hline Euzöne und tychozöne Arten & Azöne und xenozöne Arten \\
\hline Halacarellus capuzinus & Isobactrus setosus \\
Halacarellus subterraneus & Rhombognathides mucronatus \\
Anomalohalacarus tenellus & Rhombognathides pascens \\
Anomalohalacarus tenuis & Rhombognathus seahami \\
Simognathus minor & Halacarellus basteri \\
& Halacarellus longipes \\
& Agauopsis borealis \\
& Arhodeoporus submarinus \\
& Copidognathus hylandi \\
& Copidognathus novus \\
& Copidognathus punctatissimus \\
\hline
\end{tabular}


Anomalohalacarus $/ 100 \mathrm{~cm}^{3}$ wurden gezählt. Weitere Charakter-Arten des oberen und mittleren Gezeitenhanges waren Halacarellus capuzinus, $H$. subterraneus und Simognathus minor. Vereinzelt wurden auch Rhombognathinen gesehen.

Im unteren Hang war der Anteil der Anomalohalacarus-Arten unbedeutend, statt dessen dominierte Halacarellus capuzinus, der oft $20-75 \%$ der Halacariden-Fauna einnahm (vgl. Abb. 4 C). Von den übrigen, im unteren Hang angetroffenen Arten Halacarellus subterraneus, $H$. basteri, $H$. longipes, Arhodeoporus submarinus, Agauopsis borealis, Copidognathus novus, C. punctatissimus, Isobactrus setosus, Rhombognathides mucronatus, Rh. seahami, Metarhombognathus armatus - sind viele als Gäste in diesem Biotop zu bewerten.

\section{Unsortierte, grobe Sedimente}

In unsortierten, groben Sedimenten war die Halacariden-Fauna meist heterogen. Proben mit durchwurzeltem Sediment, entnommen im Hochwasserbereich eines oligobis mesomixohalinen Gewässers (Station P 2), enthielten, in jeweils geringen Zahlen, Isobactrus levis, I. setosus, Rhombognathides mucronatus, Metarhombognathus armatus, Halacarellus arenarius, $H$. balticus, $H$. capuzinus, Agauopsis borealis und Copidognathus hylandi. Halacarellus arenarius war meist die dominierende Art.

In Stränden, umspült von mesomixo- und polymixohalinem Wasser, stellte im oberen Hang Halacarellus subterraneus oft 50-90\% der Halacariden-Fauna. Starker Algen(Enteromorpha)-Bewuchs der Sedimentoberfläche jedoch führte zu einem hohen Anteil an Rhombognathinen, bedingt durch das Massenauftreten von Rhombognathides mucronatus. Auch im mittleren und unteren Hang, in dem Kiesel und Sandkörner einen reichen Algenbewuchs aufwiesen, herrschten die Rhombognathinen vor. In meso- und polyhalinen, erheblichen Salinitätsschwankungen ausgesetzten Strandabschnitten stellte Rhombognathides mucronatus oft 70-80\% der Halacariden-Fauna. In geringen Mengen erschienen Halacarellus balticus, $H$. basteri, $H$. capuzinus, Agauopsis borealis, Anomalohalacarus tenellus, Copidognathus americanus und C. hylandi.

In polymixohalinen und euhalinen Gewässerabschnitten wurden im groben, unsortierten Sediment ebenfalls viele Rhombognathinen gefunden, hier jedoch in erster Linie vertreten durch Rhombognathides pascens. An Stränden mit reichem Algenbewuchs stellte $R h$. pascens fast $83 \%$ der Halacariden-Fauna. Bei geringem Algenbewuchs betrug der Anteil der Rhombognathinen nur 20-30\%; Copidognathus-Arten, in erster Linie C. novus, und Arhodeoporus submarinus waren die wichtigsten Arten. In geringer Abundanz traten zudem Rhombognathides seahami, Halacarellus balticus, $H$. basteri, $H$. capuzinus, Copidognathus celatus, C. maculatus, C. punctatissimus und Lohmannella falcata auf.

\section{Phytal}

\section{Grünalgenpolster}

Im Enteromorpha-Band im oberen Gezeitenbereich dominierte stets Metarhombo gnathus armatus; diese Art stellte meist über $95 \%$ der Gesamtfauna und über $99 \%$ der Halacariden-Fauna (Abb. 6). An Station A 12 wurde in drei quantitativ ausgewerteter Proben 384-1573 Halacariden/10 $\mathrm{cm}^{2}$ gezählt. Die Diskrepanzen in der Siedlungsdicht $\epsilon$ wurden durch die unterschiedlichen Sandmengen, die sich zwischen den Enteromor pha-Thalli abgelagert hatten, verursacht. Neben $M$. armatus wurden vereinzelt IsobaC 
trus setosus, Rhombognathides pascens, Halacarellus capuzinus, Arhodeoporus submarinus, Copidognathus hylandi und C. novus angetroffen, wobei die Halacarellus-, Arhodeoporus- und Copidognathus-Arten wahrscheinlich eingeschwemmt worden waren.

In Enteromorpha-Proben, gesammelt im mittleren Gezeitenbereich, war die Halacariden-Fauna heterogener. Doch auch in diesem Habitat stellte Metarhombognathus armatus oft noch 73-90\% der Halacariden. In lenitischen Brackwasserregionen trat Isobactrus setosus in hoher Abundanz auf; er hatte einen Anteil von $25 \%$ an der Halacariden-Fauna. Weitere wichtige Arten der Enteromorpha-Polster waren Rhombognathides mucronatus und Halacarellus balticus.

Zwischen Enteromorpha-Thalli sedimentnaher Habitate wurde außer den Rhombognathinen auch Halacarinen - Arhodeoporus arenarius, Agauopsis borealis, Copidognathus americanus - gefunden. Unter den Rhombognathinen waren Metarhombognathus armatus und Rhombognathides mucronatus die dominanten Arten (vgl. Abb. 7A); Isobactrus setosus dagegen war selten.

Im unteren Gezeitenbereich wurde großflächiger Enteromorpha-Bewuchs nur in der Bucht bei Hamilton (Station A 1) gefunden. Am Strand wurde zwischen Enteromorpha auf Steinen und Kiesel in hoher Abundanz Arhodeoporus arenarius angetroffen (Abb. 7B), gefolgt von Metarhombognathus armatus, und, in weit geringeren Mengen, Isobactrus setosus, Rhombognathides mucronatus, Halacarellus capuzinus, Copidognathus americanus und Agauopsis borealis.

Ein Enteromorpha-Biotop, der regelmäßig erheblichen Salzgehaltsänderungen ausgesetzt ist, wurde an Station A 2 untersucht. Auch hier war Metarhombognathus armatus die dominante Art; in großen Mengen trat auch Isobactrus setosus auf; in geringen Zahlen erschienen Isobactrus hutchinsoni, Rhombognathides mucronatus, Halacarellus balticus und Copidognathus hylandi.

Zwischen den oft dichten Enteromorpha-Geflechten in Felstümpeln wurden Halacariden nur selten angetroffen.

Gering war die Halacariden-Fauna zwischen fädigen Grünalgen der Gattungen Cladophora und Chaetomorpha. Die wenigen vorgefundenen Exemplare von Metarhombognathus armatus, $M$. nudus und Rhombognathides pascens waren wahrscheinlich von benachbarten Biotopen eingeschwemmt worden.

Zwischen Monostroma, gesammelt in mesohalinen Brackwasserabschnitten an der Niedrigwasserlinie, waren Isobactrus setosus, Metarhombognathus armatus und Rhombognathides mucronatus die wichtigsten Halacariden-Arten. Im polyhalinen Brackwasserbereich fehlten meist Isobactrus setosus und Metarhombognathus armatus; in großen Mengen trat Rhombognathides pascens auf; daneben wurden $R h$. mucronatus, $R h$. seahami und Halacarellus basteri registriert.

Auf den in flachen Brackwasserbuchten angeschwemmten Ulva-Thalli dominierte Rhombognathides pascens; nur vereinzelt erschienen $R h$. mucronatus, $R h$. seahami, Metarhombognathus armatus und Copidognathus punctatissimus.

\section{Großthallige Braunalgen}

Im oberen Narragansett Bay und im Pettaquamscutt war die Halacariden-Fauna der Fucus-Thalli (F. spiralis, $F$, vesiculosus), gesammelt im oberen Gezeitenbereich, oft individuenarm, lediglich Metarhombognathus armatus wurde in einigen Proben in 
hoher Abundanz registriert (vgl. Abb. 8). Isobactrus hartmanni war regelmäßig aber in geringen Mengen vertreten; vereinzelt erschienen auch Isobactrus setosus, Rhombognathides mucronatus, Rh. pascens und Halacarellus balticus. Im mittleren und unteren Gezeitenbereich war die Halacariden-Fauna reicher, sowohl an Arten- als auch Individuenzahlen; bis zu 790 Halacariden in $1000 \mathrm{~cm}^{3}$ locker gepackten Fucus-Thalli wurden gezählt. Im mesohalinen Brackwasserbereich dominierte auf Fucus vesiculosus teils Metarhombognathus armatus - 30-96\% der Halacariden -, teils Isobactrus setosus - bis zu $54 \%$ der Halacariden; in geringen Mengen traten Rhombognathides mucronatus, $R h$. pascens, Isobactrus levis, Halacarellus balticus, $H$. capuzinus und Lohmannella falcata

Tab. 4. Halacariden-Arten auf Fucus- und Ascophyllum-Thalli gesammelt im Eulitoral



\author{
Halacarellus basteri \\ Halacarellus capuzinus \\ Halacarellus longipes \\ Arhodeoporus submarinus \\ Copidognathus maculatus \\ Copidognathus novus \\ Copidognathus punctatissimus \\ Lohmannella falcata
}

auf. Auf Fucus-Thalli, die auf sandig-kiesigen, von Spartina durchwurzelten Strandabschnitten wuchsen, dominierte Rhombognathides mucronatus; doch auch Isobactrus setosus trat in hoher Abundanz auf; Metarhombognathus armatus war mit nur wenigen Exemplaren vertreten.

In polymixohalinen und euhalinen Abschnitten, an den Stationen im unteren Narragansett Bay und am Rhode Island Sound, war die Fauna auf Fucus und Ascophyllum wesentlich individuen- und artenreicher. Im oberen Litoral wurden bis zu 1413 Halacariden, im mittleren bis zu 3023 Halacariden auf $1000 \mathrm{~cm}^{3}$ Algenthalli gefunden. Auch an diesen Stationen dominierte im mittleren Gezeitenbereich meist noch Metarhombognathus armatus (vgl. Abb. 9A, 9B); in großen Mengen traten ebenfalls Rhombognathides pascens und $R h$. seahami auf; daneben erschienen einzelne Isobactrus hartmanni, Rhombognathides mucronatus, Halacarellus basteri, H. balticus, H. longipes, Arhodeoporus submarinus, Copidognathus punctatissimus und $C$. novus. Im unteren Gezeitenbereich waren $R h$. pascens und $R h$. seahami, die dominanten Arten. An Station A 6 stellte $R h$, pascens $87 \%$ der Meeresmilben, an Station A 11 nahm Rh. seahami $62 \%$ ein, $R h$. pascens 33-34 \%. Neben diesen zwei Arten, die über $90 \%$ der HalacaridenFauna ausmachten (vgl. Abb. 9C), waren vereinzelt Isobactrus hartmanni, Isobactrus setosus, Metarhombognathus armatus, M. nudus, Halacarellus basteri, H. longipes, Arhodeoporus submarinus, Copidognathus maculatus, C. novus und Lohmannella falcata vertreten.

Die wenigen an der Niedrigwasserlinie gefundenen Laminaria-Tange waren offenbar eingeschwemmt. Die Fauna auf den Thalli war unbedeutend; zwischen den Rhizoiden aber lebte eine reiche Fauna mit Rhombognathides pascens, Rh. seahami, Halacarellus basteri, Arhodeoporus submarinus, Copidognathus celatus, C. maculatus, $C$. novus, C. punctatissimus und Lohmannella falcata. 


\section{Braun- und Rotalgenpolster im Bereich der Niedrigwasserlinie}

An Station P 7, im Übergangsbereich von polyhalinem zu mesohalinem Brackwasser, dominierte Halacarellus basteri, neben Rhombognathides pascens, Halacarellus balticus, Agauopsis borealis und Copidognathus punctatissimus.

An den Stationen am Narragansett Bay und Rhode Island Sound stellten die Rhombognathinen den größten Teil der Fauna. Auf $R h$. pascens und $R h$. seahami entfielen oft 73-96\% der Fauna; meist war Rh. pascens die dominante Art. Im Brackwasserbereich allerdings trat in flachen Strand- und Felstümpeln auch $R h$. mucronatus in hohen Zahlen auf. Metarhombognathus war selten zu sehen. Die Halacarinen und Lohmannellinen waren vertreten durch die Arten Halacarus frontiporus, Halacarellus balticus, H. basteri, H. capuzinus, H. longipes, Arhodeoporus submarinus, Copidognathus maculatus, C. novus, C. punctatissimus und Lohmannella falcata.

\section{Spaltensysteme in Tierkolonien}

\section{Balaniden-Krusten}

In den von Süßwasser und oligohalinem Brackwasser umspülten Balanus imrovisus (Station P 1) wurden viele Individuen von Copidognathus hylandi beobachtet; vereinzelt trat auch $C$. poriferus auf.

Im mesohalinen Brackwasserbereich war die Halacariden-Fauna zwischen den an und unterhalb der Niedrigwasserlinie wachsenden Balanus eburneus artenreicher. Rhombognathus notopsoides stellte 3-16\% der Fauna, Halacarellus balticus 1-82 \%, Agauopsis borealis 5-49\% und Copidognathus hylandi 10-76\%; in geringer Abundanz erschienen Metarhombognathus armatus, Rhombognathides mucronatus, Rh. pascens, Copidognathus novus und C. punctatissimus.

Zwischen den täglich erheblichen Salinitätsveränderungen ausgesetzten Balaniden der Station A 2 dominierte zahlenmäßig Halacarellus balticus (48\%), gefolgt von Metarhombognathus armatus (21\%) und Rhombognathides mucronatus (20\%); daneben wurden einzelne Exemplare von Isobactrus setosus und I. hutchinsoni den Proben entnommen.

Tab. 5. Halacariden-Arten der Balaniden-Krusten

Oligo-mesohaline Brackgewässer $(0,5-25 \% \mathrm{~S})$

Rhombognathus notopsoides Isobactrus hutchinsoni Isobactrus setosus Rhombognathides mucronatus Metarhombognathus armatus Halacarellus balticus Agauopsis borealis Copidognathus hylandi Copidognathus novus Copidognathus punctatissimus
Meso- bis polyhaline Brackgewässer $(26-33 \% \mathrm{~S})$
Isobactrus hartmanni
Isobactrus setosus
Rhombognathides mucronatus
Rhombognathides pascens
Metarhombognathus armatus
Metarhombognathus nudus
Halacarellus balticus
Arhodeoporus submarinus
Copidognathus maculatus
Copidognathus novus
Lohmannella falcata 
Zwischen Balaniden (vorwiegend Semibalanus balanoides), gesammelt im oberen Gezeitenbereich in meso- und polyhalinen Brackwässern, war die Halacariden-Fauna meist spärlich; Isobactrus hartmanni, I. setosus, Metarhombognathus armatus, Rhombognathides mucronatus, Rh. pascens und Halacarellus balticus wurden in geringer Anzahl angetroffen. Nur auf wellenexponierten Felsflächen erschien Metarhombognathus nudus in großen Mengen.

Zwischen Semibalanus balanoides-Kolonien, gesammelt im mittleren und unteren Gezeitenbereich aus meso-, poly- und euhalinen Gewässern, dominierte meist Halacarellus balticus; einen nur geringen Anteil an der Fauna hatten Isobactrus hartmanni, I. setosus, Rhombognathides mucronatus, Metarhombognathus armatus, $M$. nudus, Halacarellus basteri, Agauopsis borealis, Copidognathus americanus, C. maculatus, C. novus und Lohmannella falcata. Vereinzelt erschienen auch Rhombognathides pascens und $R h$. seahami in den Proben. Zwischen Balaniden allerdings, entnommen aus dem Enteromorpha-Streifen, beherrschte Metarhombognathus armatus zahlenmäßig die Halacariden-Fauna.

Auf einigen aus dem Sublitoral stammenden Balaniden dominierte Copidognathus punctatissimus; in geringer Abundanz traten Arhodeoporus submarinus und Copidognathus maculatus auf.

\section{Mytilus-Klumpen}

Zwischen dichten Mytilus-Klumpen, gesammelt am Narragansett Bay und Rhode Island Sound, lebten nur wenige Halacariden. Es waren einige Rhombognathides pascens, Rh. mucronatus, Metarhombognathus nudus, Halacarellus balticus und $H$. basteri.

\section{VERGLEICH DER HALACARIDEN-FAUNA AN OST- UND WESTKÜSTE DES NORDATLANTIKS}

\section{Salzwiesen}

Im oberen Puccinellietum werden im Becken von Arcachon, an der Bretagne-Küste und in der südlichen Nordsee an der SedimentoberflächeRhombognathides spinipes gefunden, auf den Sediment und Salzwiesenpflanzen überwuchernden Grünalgen Isobactrus uniscutatus (Bartsch, 1974a, 1976b, 1978). An der Bretagneküste und im Becken von Arcachon tritt im oberen Puccinellietum auch Agauopsis tricuspis auf (Bartsch, 1976b, 1978). In den stets feuchten Zonen im unteren Andelrasen und im Sediment flacher Gräben und Priele lebt - meist in hoher Abundanz - Copidognathus brevirostris; in geringen Mengen werden Rhombognathides spinipes, Agauopsis tricuspis und Halacarellus micropectinatus angetroffen (Bartsch, 1974a, 1976b, 1978). Die Charakterarten der Ostseesalzwiesen sind Halacarellus capuzinus und $H$. subterraneus; daneben erscheinen Copidognathus brevirostris, Rhombognathides spinipes und Rh. mucronatus (Bartsch, 1974a).

An den Küsten Rhode Islands ist die Fauna des Puccinellietums wesentlich artenärmer als an den Küsten Europas. Es fehlt eine an ein Leben im Hochwasserbereich spezialisierte Halacariden-Fauna. Im stets feuchten Puccinellietum und im Detritus flacher Priele dominiert beiderseits des Atlantiks eine Copidognathus-Art, nämlich 
C. brevirostris an den Küsten Europas und C. hylandi an den Küsten Rhode Islands. Die zwei Arten sind von kleiner, gedrungener Gestalt, sie sind sich im äußeren Erscheinungsbild äußerst ähnlich.

Spartina-Bestände wurden an der Nordseeküste nur in Schlicksand-Watten angetroffen. Die Halacariden-Fauna ist individuenarm; gefunden werden Isobactus setosus, I. uniscutatus, Rhombognathides spinipes, Metarhombognathus armatus, Halacarellus balticus und vor allem Copidognathus brevirostris.

An den Küsten Rhode Islands fand sich Spartina vorwiegend in detritusreichen, aber sandigen Küstenabschnitten. Während an den Nordseeküsten die Halacariden-Fauna der Spartina-Felder der des unteren Puccinellietum ähnlich ist, tritt an den Küsten Rhode Islands eine im Vergleich zum Puccinellietum andere, wesentlich arten- und individuenreichere Halacariden-Fauna auf (vgl. Tab. 2). Da die Sedimentbeschaffenheiten der Spartina-Wiesen an Ost- und Westküste des Atlantiks sehr unterschiedlich sind, ist ein direkter Vergleich nicht gegeben.

Die Abbruchkanten der Salzwiesen zu Prielen und flachen Buchten sind oft dicht mit Enteromorpha bewachsen. An den Ostseesalzwiesen leben hier vor allem Rhombognathides mucronatus und Halacarellus balticus (Bartsch, 1974a). An den Küsten Rhode Islands siedeln im Enteromorpha-Überzug ebenfalls $R h$. mucronatus und $H$. balticus, außerdem wird oft Metarhombognathus armatus in großen Mengen registriert.

\section{Sandstrände polymixohaliner und euhaliner Gewässer}

Ergebnisse qualitativer und quantitativer Untersuchungen liegen von Sandstränden vor Roscoff (Bartsch, 1978, 1979a) und Sylt (Bartsch \& Schmidt, 1979) vor. In mittellotischen Stränden auf Sylt sind die wichtigsten Arten Rhombognathus intermedius, Actacarus pygmaeus, Halacarellus capuzinus, H. procerus, $H$. subterraneus, Anomalohalacarus minutus und $A$. arenarius. Die Anomalohalacarus-Arten sind im gesamten Hang verbreitet; Actacarus pymaeus dominiert in der Feuchtsandzone im mittleren Hang; Halacarellus procerus bevorzugt meist den mittleren Hang und $H$. capuzinus den unteren Hang; Rhombognathus intermedius wird in großen Mengen im stets wassergesättigten Sandwatt gefunden. An der Bretagne-Küste leben in sortierten Mittelsanden Rhombognathus intermedius, Rhombognathides merrimani, Actacarus pygmaeus, Halacarellus capuzinus und Acaromantis minutus; in groben Sedimenten treten Anomalohalacarus-Arten (arenarius, intermedius, minutus), Halacarellus capuzinus, $H$. procerus und Copidognathus rhodostigma auf. Im oberen Hang dominiert Rh. merrimani, an zweiter Stelle steht Anomalohalacarus minutus. Im mittleren und unteren Hang herrscht Rhombognathus intermedius vor, daneben erscheinen Rhombognathides merrimani, Halacarellus procerus, $H$. capuzinus, die Anomalohalacarus-Arten und Copidognathus rhodostigma. In Brackwasser-beeinflußten Stränden des Penzé-Aestuars sind Rhombognathides spinipes und Halacarellus capuzinus die wichtigsten Arten (Bartsch, 1978, 1979a).

Vertreter der Gattung Actacarus sind bisher an Gezeitenstränden Nordostamerikas nicht nachgewiesen. Auch eine psammobionte Rhombognathus-Art, entsprechend der europäischen $R h$. intermedius, ist nicht bekannt. In unsortierten Sedimenten an Brackwasserstränden erscheinen unter den Rhombognathinen an den Küsten Nordostamerikas vor allem Rhombognathides mucronatus, an den Küsten Nordeuropas Rhombogna- 
thides merrimani und $R h$. spinipes. $R h$. merrimani ist vom Norden der amerikanischen Ostküste, von New Brunswick gemeldet (Newell, 1947), doch ist nicht bekannt, welche Biotope die Art dort besiedelt. Die Gattung Acaromantis ist an den beiden Küsten mit je einer Art vertreten. An den Küsten Rhode Islands tritt zudem eine Simognathus-Art auf; an den Küsten Nordeuropas leben Arten dieser Gattung vorwiegend an und unterhalb der Niedrigwasserlinie. An beiden Seiten des Atlantiks siedeln Halacarellus capuzinus und $H$. subterraneus; es werden jeweils vergleichbare Abschnitte im Hang bevorzugt. Eine dritte an den Küsten Europas im Mesopsammal verbreitete Halacarellus-Art, $H$. procerus, wurde nicht in den untersuchten Stränden Rhode Islands angetroffen; die Art ist jedoch von Stränden Massachusetts gemeldet (Bartsch, 1976a). In Gezeitenstränden Nordamerikas treten von der Gattung Anomalohalacarus zwei Arten auf, an den Küsten des borealen Europas drei Arten. Die Gattung Copidognathus ist erst im unteren Hang und in Sandwatten präsent. An der Bretagneküste sind vor allem C. rhodostigma und C. venustus, vereinzelt auch $C$. brevirostris zu finden; von den Küsten Rhode Islands sind C. americanus und - seltener beobachtet - C. hylandi zu nennen. C. americanus ist $C$. rhodostigma im Habitus äußerst ähnlich; die zwei Arten nehmen jeweils auf ihrer Seite des Atlantiks einen entsprechenden Lebensraum ein. In wesentlich höherer Abundanz als an den Küsten Europas ist an den Rhode Islands die Gattung Arhodeoporus vertreten. Die amerikanischen Arten A. arenarius und A. submarinus besiedeln feuchte Sandwatten und das Sublitoral; die europäische Art $A$. gracilipes aber ist in erster Linie ein Bewohner sublitoraler Sedimente.

Die Halacariden-Fauna der Gezeitenstrände Nordostamerikas ist - nach den jetzigen Kenntnissen - etwas artenärmer als die Nordeuropas.

\section{Grünalgenzone}

Zwischen Enteromorpha im oberen und mittleren Gezeitenbereich polymixohaliner und euhaliner Küstenabschnitte dominiert an den Küsten Europas meist Metarhombognathus armatus, wiederholt wird auch Halacarellus balticus angetroffen (Bartsch, 1974a, 1978); an einigen Orten tritt Isobactrus setosus und I. hartmanni in großen Mengen auf.

An den Küsten Rhode Islands war nur an wenigen Stellen ein breiter EnteromorphaStreifen entwickelt. Auch hier dominiert Metarhombognathus armatus; in stets feuchten Polstern ist auch Halacarellus balticus zu sehen. Die Halacariden-Fauna der Enteromorpha-Zone ist also beiderseits des Atlantiks sehr ähnlich.

Im oligohalinen Brackwasserbereich siedelt an den Küsten Nordeuropas im Enteromorpha-Streifen Isobactrus uniscutatus, eine Art, die nicht nur an niedrige Salinität, sondern auch an langanhaltende Trockenphasen angepaßt ist (Bartsch, 1974a). An den Küsten Nordostamerikas tritt die $I$, uniscutatus nahverwandte Art $I$. hutchinsoni auf. $I$. hutchinsoni hat, wie I. uniscutatus, den oligohalinen Brackwasserbereich erobert, er verfügt aber nicht über die für $I$. uniscutatus so charakteristische Austrocknungsresistenz.

An den europäischen Küsten ist in der Monostroma-Zone Isobactrus setosus in hoher Individuendichte vorhanden. Auch an den Küsten Rhode Islands steht im mesohalinen Brackwasserbereich Isobactrus setosus an erster Stelle; im polyhalinen Bereich ist vor allem Metarhombognathus armatus stark vertreten. 


\section{Braunalgen-Tange}

An den Küsten der südlichen Nordsee werden von Fucus vesiculosus aus lenitischen Lebensräumen große Mengen an Isobactrus setosus, aus mittellotischen Zonen Metarhombognathus armatus und aus lotischen Küstenabschnitten Rhombognathides seahami und Rh, pascens gemeldet; auf Fucus an Faschinen von Lahnungsfeldern erscheint auch Rhombognathides spinipes (Bartsch, 1974a). Im unteren Eulitoral werden regelmäBig auch Halacarellus basteri und $H$. balticus angetroffen. An der Bretagneküste besiedeln wesentlich mehr Arten die Braunalgen-Tange (Fucus, Ascophyllum) (vgl, Bartsch, 1978, 1979a), viele aber nur als Gästé. Außer den für den Nordseeraum genannten Arten ŝreten regelmäßig Rhombognathides merrimani und Rhombognathus notops auf. Auf den langen Trockenzeiten ausgesetzten Thalli im oberen Gezeitenbereich dominiert oft Isobactrus hartmanni (Bartsch, 1978).

Die Halacariden-Fauna der Fucus- und Ascophyllum-Thalli ist auf beiden Seiten des Atlantiks ähnlich, meist sind dieselben Arten dominant, doch ist die Artenzahl an den Küsten Rhode Islands deutlich geringer als an der Bretagneküste.

\section{Büschelige, reich-verzweigte Algen an der Niedrigwasserlinie}

An der Bretagneküste leben zwischen Kleinalgen (Chondrus, Cystoseira, Corallina) an der Niedrigwassergrenze Rhombognathus notops, Rhombognathides pascens, Rh. seahami, Copidognathus oculatus, daneben erscheinen in geringer Abundanz Halacarellus basteri, H. striatus, Halacarus actenos, Arhodeoporus gracilipes, Agauopsis brevipalpus, Copidognathus fabricii, C. figeus, C. remipes, C. pseudofigeus und Lohmannella kervillei (Bartsch, 1978).

Zwei der wichtigsten Arten sowohl im Ost- als auch Westatlantik sind Rh. pascens und $R h$. seahami. Im Ostatlantik aber dominiert im Gezeitenbereich stets $R h$. seahami; $R h$. pascens wird in großen Mengen nur auf ständig wasserbedeckten Thalli gefunden. An den Küsten Rhode Islands aber wurden nur in wenigen Proben mehr $R h$. seahami als $R h$. pascens gezählt; $R h$. pascens besiedelte ein weit größeres Areal als $R h$. seahami, war regelmäßig im Eulitoral präsent und übertraf zahlenmäßig meist $R h$. seahami. Eine Rhombognathus-Art, die die Stelle von $R h$. notops einnehmen könnte, ist im untersuchien Raum der USA nicht gefunden worden. Halacarellus basteri, $H$. balticus und einzelne $H$. capuzinus werden an beiden Küsten angetroffen. Zwischen den büscheligen Algen der Niedrigwasserlinie treten stets mehrere Copidognathus-Arten auf, doch hat jede Küste ihre eigenen Arten. Lohmannella kervillei wird an den Küsten Rhode Islands durch $L$. falcata ersetzt.

\section{Balaniden-Krusten}

Die Halacariden-Fauna der Balaniden-Krusten ist von Standort und Algenbewuchs abhängig. In der südlichen Nordsee wird im meso- und polyhalinen Brackwasserbereich Halacarellus balticus in großer Individuendichte angetroffen (Bartsch, 1974a). Auf dem Grünalgenbewuchs der Balaniden treten Rhombognathinen auf; auf sonnenexponierten Stellen im oberen und mittleren Gezeitenbereich dominieren Isobactrus uniscutatus und I. hartmanni, in stets feuchten, ruhigen Zonen Isobactrus setosus, an wellen-exponierten Flächen Metarhombognathus armatus (Bartsch, 1974a und unveröff. Ergebnisse). An der 
Bretagneküste sind im Fluß Penzé im mesohalinen Bereich Isobactrus uniscutatus, I. setosus und Metarhombognathus armatus verbreitet; im polyhalinen Bereich leben auf Elminius modestus Isobactrus ungulatus, I. rugosus, I. setosus, Copidognathus figeus und Agauopsis tricuspis; in marinen Gewässern an der Küste werden im oberen Gezeitenbereich Isobactrus rugosus, einzelne I. pulchellus und Agauopsis tricuspis beobachtet.

Die Fauna der Balaniden-Krusten wird beiderseits des Atlantiks im meso- und polyhalinen Bereich bestimmt durch die Arten Metarhombognathus armatus, Isobactrus setosus, I. hartmanni und Halacarellus balticus. Nicht von der südlichen Nordsee, wohl aber von deren nördlichen Teil ist Metarhombognathus nudus bekannt. Eine an oligohaline Bedingungen angepaßte Balaniden-Fauna, wie sie im borealen Westatlantik verbreitet ist (vgl. Poirrier \& Partridge, 1979) fehlt in den bisher untersuchten Aestuaren der Nordsee und der Ostsee.

\section{VERBREITUNG DER HALACARIDEN IN DER ATLANTISCH-BOREALEN ZONE}

Bisher sind etwa 500 Halacariden-Arten beschrieben. Aus dem Nordostatlantik, vom Englischen Kanal bis nach Nordnorwegen, die Ostsee eingeschlossen, sind 91 Arten gemeldet, aus dem Bereich des Englischen Kanals 74 Arten, aus dem Nordseeraum 51 Arten. Dagegen sind vom Nordostatlantik, nördlich von Kap Hatteras, nur 38 Arten bekannt (Tab. 1). Allerdings muß berücksichtigt werden, daß die HalacaridenFauna an den Küsten der USA bei weitem weniger untersucht ist als die der europäischen Küsten. $\mathrm{Ob}$ die Zahl der Arten an den Küsten des Nordwestatlantiks wirklich unter der des Nordostatlantiks liegt (vgl. Briggs, 1974), kann erst nach weiteren Probenentnahmen beurteilt werden.

Tabelle 6 gibt eine Übersicht über die an der Küste Nordamerikas, nördlich von Kap Hatteras, gefundenen Arten; die Tabelle gibt auch Auskunft über Vorkommen bzw. Fehlen der Arten an den Küsten Europas. Von den 38 Arten sind 17, also 45 \%, auch an den Küsten Europas beheimatet. Der prozentuale Anteil amphiatlantischer Arten ist somit etwas geringer als von Newell (1947) angenommen.

Wie ist die amphiatlantische Verbreitung zu erklären? Halacariden sind Benthosorganismen. Planktonformen oder im Plankton lebende Entwicklungsstadien fehlen; die Jugendstadien leben ähnlich wie die Adulten, sie besiedeln denselben Lebensraum. Größere Strecken über Meeresarme oder Meere hinweg können nur durch passiven Transport bewältigt werden, durch Wanderung oder Verdriftung der besiedelten Tiere und Algen und durch Verschleppung durch den Menschen. Ein passiver Transport mit dem besiedelten Substrat - über den Atlantik hinweg - wäre von Amerika nach Europa auf dem Golfstrom möglich, von Europa nach Amerika - besonders im Jungtertiär oder Pleistozän - entlang einer Kette von Inseln im Bereich des Färöer-Island-GrönlandRückens (Lindroth, 1957, 1963; Donn et al. 1962; Strauch, 1970). Eine passive Verschleppung mit Schiffen wäre in erster Linie durch Ballast (Steine, Sand, Wasser), außerdem auch im Bewuchs der Schiffe denkbar.

Beeinflußt oder begünstigt die Lebensweise eine amphiatlantische Verbreitung? Es wäre zu erwarten, daß für Arten, die im Bereich der Niedrigwasserlinie oder im flachen Sublitoral leben, ohne strenge Bindung an ein bestimmtes Substrat, die Chancen groß 
sind, einen Wochen oder Monate dauernden Transport zu überstehen und an neuen Küsten einen Siedlungsraum zu finden. Arten dagegen, die sich an die besonderen Lebensbedingungen der Biotope Brackwasser, oberes und mittleres Eulitoral oder Sand-

Tab. 6. Halacariden-Arten gefunden an der Ostküste Amerikas nördlich von Kap Hatteras, mit Hinweisen zum Vorkommen $(+)$ oder Fehlen $(-)$ an den Küsten Europas

\begin{tabular}{|c|c|c|}
\hline Species & USA & Europa \\
\hline Rhombognathus notopsoides & + & _- \\
\hline Rhombognathus sp. & + & - \\
\hline Isobactrus hartmanni & + & + \\
\hline Isobactrus hutchinsoni & + & - \\
\hline Isobactrus levis & + & + \\
\hline Isobactrus setosus & + & + \\
\hline Rhombognathides merrimani & + & + \\
\hline Rhombognathides mucronatus & + & + \\
\hline Rhombognathides pascens & + & + \\
\hline Rhombognathides seahami & + & + \\
\hline Metarhombognathus armatus & + & + \\
\hline Metarhombognathus nudus & + & + \\
\hline Halacarus frontiporus & + & - \\
\hline Halacarellus arenarius & + & - \\
\hline Halacarellus balticus & + & + \\
\hline Halacarellus basteri & + & + \\
\hline Halacarellus capuzinus & + & + \\
\hline Halacarellus dissimilis & + & - \\
\hline Halacarellus longipes & + & + \\
\hline Halacarellus procerus & + & + \\
\hline Halacarellus subterraneus & + & + \\
\hline Agauopsis borealis & + & - \\
\hline Arhodeoporus arenarius & + & - \\
\hline Arhodeoporus submarinus & + & - \\
\hline Anomalohalacarus litoralis & + & - \\
\hline Anomalohalacarus tenellus & + & - \\
\hline Anomalohalacarus tenuis & + & - \\
\hline Copidognathus americanus & + & - \\
\hline Copidognathus celatus & + & - \\
\hline Copidognathus hylandi & + & - \\
\hline Copidognathus maculatus & + & - \\
\hline Copidognathus novus & + & - \\
\hline Copidognathus poriferus & + & - \\
\hline Copidognathus punctatissimus & + & - \\
\hline Copidoqnathus pygmaeus & + & - \\
\hline Lohmannella falcata & + & + \\
\hline Simognathus minor & + & - \\
\hline Acaromantis arenarius & + & - \\
\hline
\end{tabular}

lückensystem angepaßt haben, werden kaum Aussichten haben, einen längeren Transport bei hoher Salinität, ständiger Überflutung bzw. dem Fehlen eines geeigneten Mesopsammals zu überleben und in einen geeigneten Lebensraum gespült zu werden.

Tabelle 7 gibt eine Liste der an der Ostküste Nordamerikas gefundenen Arten 
Tab. 7. Halacariden-Arten im borealen Nordostamerika mit Hinweisen zu Biotopbedingungen und amphiatlantischer Verbreitung: + regelmäßig, meist in großen Mengen, in diesem Lebensraum gefunden; a amphiatlantische Art; - nur vom amerikanischen Kontinent bekannt

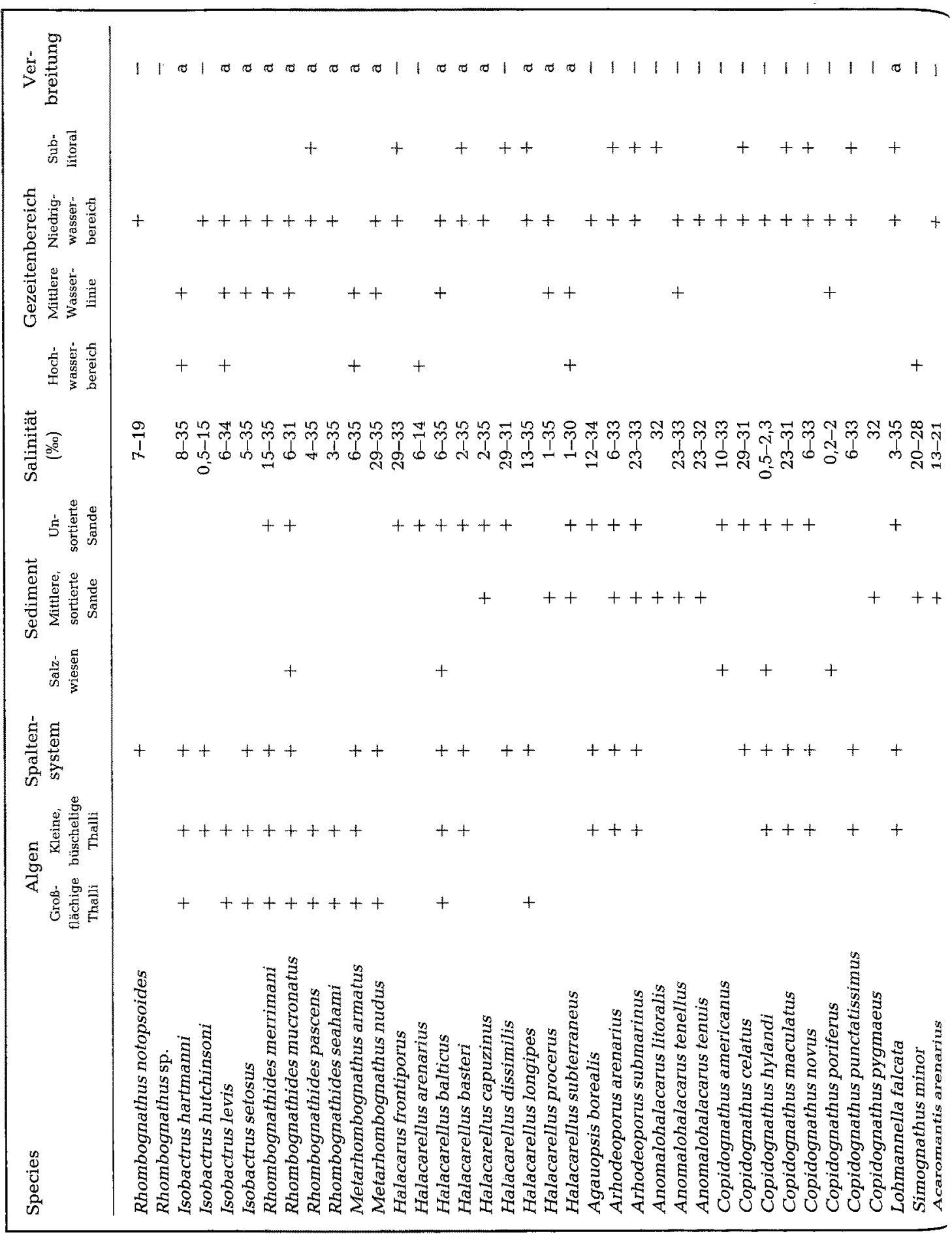


wieder mit Hinweisen zu den Lebensräumen, in denen sie an den Küsten Nordostamerikas und Europas angetroffen werden. Faktoren, die die Besiedlung der Halacariden beeinflussen, sind Substrat, Salinität und Dauer der Uberflutung (Tidenbereich). In der Rubrik Substrat wird unterschieden zwischen Algen (kleinflächige, büschelige Algen und Laminaria-Rhizoide; großthallige Algen), Spaltensystemen zwischen Tierkolonien (Bryozoen, Balaniden, Mytilus) und Sedimenten (Salzwiesenböden; sortierte, mittelfeine Sande; unsortierte Sande). In der Liste werden in erster Linie die Biotope genannt, in denen die Arten regelmäßig, oft in großen Mengen, gefunden werden; Einwanderer aus benachbarten Lebensräumen werden nicht berücksichtigt. Bei Arten, von denen visher nur wenige Exemplare bekannt sind, mußte allerdings auf diese wenigen Fundorte verwiesen werden.

Für die Bewohner der drei Lebensräume Brackwasser, oberer und mittlerer Gezeitenbereich und Sandlückensystem könnte angenommen werden, daß der Atlantik eine unüberbrückbare Barriere darstellt.

$\mathrm{Zu}$ den Brackwassertieren werden Arten gezählt, die ausschließlich in Brackwasser unter $25 \% \mathrm{~S}$ vorkommen, in Gewässern mit höherer Salinität aber fehlen. Brackwasserarten sind - nach den bisherigen Kenntnissen - Rhombognathus notopsoides, Isobactrus hutchinsoni, Halacarellus arenarius, Copidognathus hylandi und C. poriferus. Diese Arten sind ausschließlich in Amerika beheimatet. An den Küsten Europas sind vergleichbare Biotope durch andere Arten besetzt. Im Elbe-Aestuar lebt im stark ausgesüßten Bereich Rhombognathus cetratus. Statt Isobactrus hutchinsoni wird in den Tidegewässern Europas $I$. uniscutatus gefunden. Halacarellus arenarius ist verwandt mit $H$. subterraneus. In einem Lebensraum wie er von Copidognathus hylandi besiedelt wird, ist an den Küsten Europas Copidognathus brevirostris verbreitet. $C$. poriferus ist an ein Leben in sehr stark ausgesüßtem Brackwasser angepaßt, was sich morphologisch durch die stark vergrößerten Epimeralporen, dem Ort der Ionenregulation bei CopidognathusArten (vgl. Bartsch, 1974a, 1974b), bemerkbar macht. Von den Küsten Nordeuropas ist aus der oligohalinen Brackwasserzone keine Copidognathus-Art bekannt; in Gewässern Bulgariens und Jugoslawiens aber lebt C. tectiporus, ebenfalls ausgerüstet mit stark vergrößerten Epimeralporen (Viets, 1935).

An den Küsten Rhode Islands wurde im oberen Tidenbereich auf Hartsubstrat nur an wenigen Orten ein als Siedlungsraum für Halacariden geeigneter Bewuchs gefunien; so sind auch keine Halacariden-Arten bekannt, die ausschließlich oder vorwiegend in der Hochwasserzone beheimatet sind. Die in Tabelle 7 genannten Arten sind stets auch in tiefer liegenden Regionen zu finden. Charakterarten des mittleren Gezeitenbereichs sind Isobactrus hartmanni, I setosus, Metarhombognathus armatus und M. nudus. Diese Arten sind auch von Küsten Europas gemeldet.

Charakterarten sortierter Sande im Gezeitenbereich sind Halacarellus capuzinus, H. procerus, H. subterraneus, Anomalohalacarus tenellus, A. tenuis und Simognathus minor. Die drei Halacarellus-Arten sind auch an den Küsten Europas verbreitet. Von der Gattung Anomalohalacarus treten in Gezeitenstränden Nordeuropas drei nahverwandte Arten auf (A. arenarius, $A$. intermedius, $A$. minutus). Die Gattung Simognathus ist aus dem Ostatlantik nur aus stets überfluteten Lebensräumen bekannt; doch entsendet die abenfalls zur Unterfamilie der Simognathinae gehörende Gattung Acaromantis einen Vertreter in Gezeitenstrände.

Für Bewohner vieler Lebensräume im unteren Eulitoral und flachen Sublitoral 
beständen Aussichten, mit dem Substrat losgerissen und verdriftet zu werden, dann einen langen Transport $\mathrm{zu}$ überdauern und einen neuen Lebensraum $\mathrm{zu}$ finden. Tabelle 7 zeigt, daß mehrere Arten (Halacarellus basteri, H. longipes, Lohmannella falcata) aus dem unteren Eulitoral amphiatlantisch leben, andere Arten dagegen, so die der Gattungen Arhodeoporus und Copidognathus ausschließlich von der amerikanischen Küste bekannt sind.

Die Untersuchungen der Halacariden-Fauna beiderseits des Atlantiks weisen darauf hin, daß - abgesehen vom Biotop ausgesüßtes Brackwasser - keine direkten Beziehungen zwischen Lebensraum und amphiatlantischer Verbreitung bestehen. Auswertungen von Planktonproben und treibenden Algen ergaben, daß Halacariden wohl in Küstennähe, kaum aber küstenfern gefunden werden (Lohmann, 1893); bisher ist nur ein Exemplar weit entfernt von der nächsten Küste aus Tiefenwasser bekannt geworden (Lohmann, 1893). Auf treibenden Sargassum-Tangen wurden keine Halacariden registriert (Lohmann, 1893; eigene Untersuchungen). Die bisherigen Untersuchungen zeigen, daß über weite Wasserflächen hinweg ein passiver Transport auf treibenden AlgenFlößen sehr unwahrscheinlich ist.

Eine amphiatlantische, ja sogar weltweite Verbreitung ist auch von anderen Milben bekannt, für die eine aktive Wanderung oder passive Verbreitung selbst über enge Meeresarme hinweg fast auszuschließen ist. Die in der Nähe kalter Quellen lebende Oribatei Mucronothrus nasalis ist von Grönland, Südamerika und Neuseeland gemeldet (Hammer, 1965). Mehrere der in Süßwasser lebenden und auf diesen Lebensraum angewiesenen Halacariden sind in Europa, Asien, Afrika und Amerika verbreitet (Bartsch, im Druck). Von einigen Gattungen der Oribatei sind jeweils zu einer Verwandtschaftsgruppe gehörende Arten auf mehreren Kontinenten nachgewiesen (Hammer \& Wallwork, 1979). Die Verbreitungsbilder deuten darauf hin, daß viele dieser weltweit verbreiteten Arten oder Verwandtschaftsgruppen Relikte einer Fauna sind, die im Mesozoikum die große Kontinentalplatte Pangäa besiedelten (Hammer \& Wallwork, 1979; Bartsch, im Druck).

Wie Tabelle $6 \mathrm{zu}$ entnehmen ist, sind unter den marinen Halacariden amphiatlantische Arten auf wenige Gattungen beschränkt, nämlich auf die Gattungen Isobactrus, Rhombognathides, Metarhombognathus und Halacarellus. Innerhalb der Gattungen Arhodeoporus, Anomalohalacarus und Copidognathus dagegen tritt keine Art beiderseits des Atlantiks auf. Viele der amphiatlantischen Arten der Gattungen Isobactrus, Rhombognathides, Metarhombognathus und Halacarellus sind offenbar in die Gruppe jener Milben einzureihen, die ein geologisch hohes Alter haben. Es ist zu vermuten, daß diese Arten bereits zur Zeit der letzten Trennung der amerikanischen und europäischen Kontinentalplatten, also vor rund 100 und mehr Millionen Jahren, die Küsten des Atlantischen Ozeans besiedelten. Tabelle 7 zeigt, daß amphiatlantische Arten ein weites Salinitätsspektrum einnehmen. Hinweise auf Vorkommen dieser Arten außerhalb der borealen Klimazone weisen darauf hin, daß Populationen dieser Arten auch gegenüber hohen und niedrigen Temperaturen unempfindlich sind. Die amphiatlantischen Arten haben offenbar - dank hoher Euryvalenz - die wechselnden Umweltbedingungen während vieler Millionen Jahre unverändert überdauert. Auch die Gattungen Rhombognathus, Halacarus, Arhodeoporus, Agauopsis und Anomalohalacarus sind weltweit verbreitet (vgl. Bartsch \& Schmidt, 1978; Bartsch, im Druck), auch diesen Gattungen kommt sicherlich ein hohes Alter zu. Das geographisch geringe Ausbreitungsareal der 
einzelnen Arten aber weist darauf hin, daß Änderungen der Umweltbedingungen, ökologische Nischen und geographische Barrieren zur Speziation führten.

Danksagung. Der Aufenthalt an der University of Rhode Island wurde durch ein Stipendium der Deutschen Forschungsgemeinschaft (Ba 615/1) finanziert, das Auswerten der gesammelten Proben unterstützt durch eine Sachbeihilfe (Ba 615/2). Prof. K. E. Hyland und Dr. M. G. Kraus danke ich für die unermüdliche Hilfe und Unterstützung in Rhode Island.

\section{ZITIERTE LITERATUR}

Bartsch, I., 1972. Ein Beitrag zur Systematik, Biologie und Ökologie der Halacaridae (Acari) aus dem Litoral der Nord- und Ostsee. I. Systematik und Biologie. - Abh. Verh. naturw. Ver. Hamburg 16, 155-230.

Bartsch, I., 1974a. Ein Beitrag zur Systematik, Biologie und Ökologie der Halacaridae (Acari) aus dem Litoral der Nord- und Ostsee. II. Ökologische Analyse der Halacaridenfauna. - Abh. Verh. naturw. Ver. Hamburg 17, 9-53.

Bartsch, I., 1974b. Über das Auftreten von Epimeralporen besonders bei den Rhombognathinen (Halacaridae, Acari). - Zool. Anz. 193, 266-268.

Bartsch, I., 1976a. Zur Systematik und Verbreitung der Halacarellus-Arten (Halacaridae, Acari) an der Bretagne-Küste. - Ent. Mitt. zool. Mus. Hamburg 5 (94), 97-109.

Bartsch, I., 1976b. Ergänzungen zur Halacariden-Fauna (Halacaridae, Acari) im Becken von Arcachon. - Vie Milieu, 26 (A) 31-46.

Bartsch, I., 1978. Verbreitung der Halacaridae (Acari) im Gezeitenbereich der Bretagne-Küste, eine ökologische Analyse. I. Verbreitung der Halacariden. - Cah. Biol. mar. 19, 363-383.

Bartsch, I., 1979a. Verbreitung der Halacaridae (Acari) im Gezeitenbereich der Bretagne-Küste, eine ökologische Analyse. II. Quantitative Untersuchungen und Faunenanalyse. - Cah. Biol. mar. 20, 1-28.

Bartsch, I., 1979b. Halacaridae (Acari) von der Atlantikküste Nordamerikas. Beschreibung der Arten. - Mikrofauna Meeresboden 79, 1-62.

Bartsch, I., 1980. Halacaridae (Acari) im marinen Mesopsammal der Ostküste Nordamerikas. - Ent. Mitt. zool. Mus. Hamburg $6(110), 393-407$.

Bartsch, I., 1981a. Die Gattungen Anomalohalacarus und Halacaroides (Halacaridae, Acari), geographische Verbreitung, Bestimmungstabelle und Beschreibung drei neuer Arten. - Zool. Beitr. 27, 67-84.

Bartsch, I., 1981b, Halacaridae (Acari) im Süßwasser von Rhode Island, USA, mit einer Diskussion über Verbreitung und Abstammung der Halacaridae. - Gewäss. Abwäss. (im Druck).

Bartsch, I. \& Schmidt, P., 1978. Interstitielle Fauna von Galapagos, XXII, Zur Ókologie der Halacaridae (Acari). - Mikrofauna Meeresboden 69, 1-37.

Bartsch, I. \& Schmidt, P., 1979. Zur Verbreitung und Okologie einiger Halacaridae (Acari) in Sandstränden der Ostsee (Kieler Bucht), der Nordsee (Sylt) und des Europäischen Nordmeeres (Tromsö). - Mikrofauna Meeresboden 74, 1-37.

Briggs, J. C., 1974. Marine zoogeography. McGraw-Hill, New York, 475 pp.

Collins, B. P., 1976. Suspended material transport: Narragansett Bay Area, Rhode Island. - Estuar. coast. mar. Sci. 4, 33-44.

Donn, W. L., Farrand, W. R. \& Ewing, M., 1962. Pleistocene ice volumes and sea-level lowering. J. Geol. 70, 206-214.

Halvorson, W. L. \& Dawson, C. G., 1973. Coastal vegetation. In: Coastal and offshore environmental inventory Cape Hatteras to Nantucket Shoals. - Univ. Rhode Island mar. Publ. Ser. 3 (9), 1-92.

Hammer, M., 1965. Are low temperatures a species-preserving factor? nllustrated by the oribatid mite Mucronothrus nasalis (Willm.) - Acta Univ. lund. (Sect. 2) 1965 (2), 1-10.

Hammer, M. \& Wallwork, J. A.,1979. A review of the world distribution of oribatid mites (Acari: Cryptostigmata) in relation to continental drift. - Biol. Skr. $22(4), 1-31$.

Haq, J., 1965. Records on some interstitial mites from Nobska Beach together with a description of

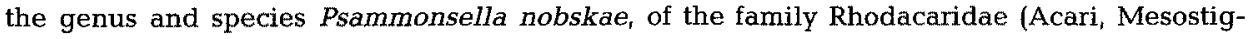
mata). - Acarologia 7, 411-419. 
Hicks, S. D., 1963. Physical oceanographic studies of Narragansett Bay 1957 and 1958. - Spec. scient. Rep. U. S. Fish Wildl. Serv. (Fisheries) 457, 1-30.

Kraus, M. G., 1980. Factors correlated with intertidal distribution of meiofauna along a Rhode Island estuary. Ph. D. Diss., University of Rhode Island, Kingston, R. I.

Kremer, J. N. \& Nixon, S. W., 1978. A coastal marine ecosystem. Simulation and analysis. - Ecol. Stud. 24, 1-217.

Lindroth, C. H., 1957. The faunal connections between Europe and North America. - Almquist \& Wiksell, Stockholm, 344 pp.

Lindroth, C. H., 1963. The problem of late land connections in the North Atlantic area. In: North Atlantic and their history. Ed. by A. Löve \& D. Löve. Pergamon Press, Oxford, 73-85.

Lohmann, H., 1893. Die Halacarinen der Plankton-Expedition. - Ergebn. Atlant. Ozean Planktonexped. Humboldt-Stift. 2 G. a. B, 11-95.

McCaffrey, R. J., Myers, A. C., Davey, E., Morrison, G., Bender, K., Luedtke, N., Cullen, D., Froelich, P. \& Klinkhammer, G., 1980. The relation between the pore water chemistry and benthic fluxes of nutrients and manganese in Narragansett Bay, Rhode Island, - Limnol. Oceanogr. 25, $31-44$.

Milliman, J. D., 1973. Marine Geology. - In: Coastal and offshore environmental inventory Cape Hatteras to Nantucket shoals. - Univ. Rhode Island mar. Publ. Ser. 3 (10), 1-91.

Newell, I. M., 1947. A systematic and ecological study of Halacaridae of eastern North America. Bull. Bingham oceanogr. Coll. 10, 1-232.

Newell, I. M., 1949. New genera and species of Halacaridae (Acari). - Am. Mus. Novit. 1411, 1-22.

Poirrier, M. A. \& Partridge, M. R., 1979. The barnacle, Balanus subalbidus, as a salinity bioindicator in the oligohaline estuarine zone. - Estuaries 2, 204-206.

Pratt, S. D., 1973. Benthic fauna. In: Coastal and offshore environmental inventory Cape Hatteras to Nantucket Shoals. - Univ. Rhode Island mar. Publ. Ser. 2 (5), 1-70.

Stickney, A. P. \& Stricker, L. D., 1957. A study of the invertebrate bottom fauna of Greenwich Bay, Rhode Island. - Ecology 38, 111-122.

Strauch, F, 1970. Die Thule-Landbrücke als Wanderweg und Faunenscheide zwischen Atlantik und Skandik im Tertiär. - Geol. Rdsch. 60, 381-417.

Viets, K., 1935. Wassermilben aus Bulgarien, - Zool. Anz. 109, 33-39. 